\title{
Recent Advances in Phase-Sensitive Optical Time Domain Reflectometry (Ф-OTDR)
}

\author{
Yunjiang $\mathrm{RAO}^{1,2,3^{*}}$, Zinan $\mathrm{WANG}^{1 *}$, Huijuan $\mathrm{WU}^{1}$, Zengling $\mathrm{RAN}^{1,3}$, and Bing $\mathrm{HAN}^{1}$ \\ ${ }^{1}$ Fiber Optics Research Center (FORC), Key Lab of Optical Fiber Sensing \& Communications, University of Electronic \\ Science \&Technology of China, Chengdu 611731, China \\ ${ }^{2}$ Research Center for Optical Fiber Sensing, Zhejiang Laboratory, Hangzhou 310000, China \\ ${ }^{3}$ PetroChina Optical Science \& Technology (Chengdu) Ltd., Chengdu 611731, China \\ *Corresponding author: Yunjiang RAO and_ E-mail: yjrao@uestc.edu.cn and \\ Zinan WANG znwang@uestc.edu.cn
}

\begin{abstract}
Phase-sensitive optical time domain reflectometry ( $\Phi-O T D R)$ is an effective way to detect vibrations and acoustic waves with high sensitivity, by interrogating coherent Rayleigh backscattering light in sensing fiber. In particular, fiber-optic distributed acoustic sensing (DAS) based on the $\Phi-O T D R$ with phase demodulation has been extensively studied and widely used in intrusion detection, borehole seismic acquisition, structure health monitoring, etc., in recent years, with superior advantages such as long sensing range, fast response speed, wide sensing bandwidth, low operation cost and long service lifetime. Significant advances in research and development (R\&D) of $\Phi-O T D R$ have been made since 2014. In this review, we present a historical review of $\Phi-O T D R$ and then summarize the recent progress of $\Phi-O T D R$ in the Fiber Optics Research Center (FORC) at University of Electronic Science and Technology of China (UESTC), which is the first group to carry out R\&D of $\Phi-O T D R$ and invent ultra-sensitive DAS (uDAS) seismometer in China which is elected as one of the ten most significant technology advances of PetroChina in 2019. It can be seen that the $\Phi-O T D R / D A S$ technology is currently under its rapid development stage and would reach its climax in the next 5 years.
\end{abstract}

Keywords: Distributed optical fiber sensing; phase-sensitive optical time domain reflectometry; Rayleigh backscattering; optical phase demodulation; distributed acoustic sensing; long-distance sensing; artificial intelligence

Citation: Yunjiang RAO, Zinan WANG, Huijuan WU, Zengling RAN, and Bing HAN, "Recent Advances in Phase-Sensitive Optical Time Domain Reflectometry (Ф-OTDR),” Photonic Sensors, 2021, 11(1): 1-30.

\section{Operation principle of $\Phi$-OTDR}

In a phase-sensitive optical time domain reflectometry ( $\Phi-O T D R)$, the narrow linewidth pulse light is injected into the sensing fiber through the circulator. Then, the Rayleigh backscattering signal carrying the sensing information is received by the photodetector (PD). The theoretical model of the vibration detection principle based on $\Phi-O T D R$ is established as following.

The incident lightwave field $E$ of $\Phi$-OTDR can be expressed as

$$
E=E_{0} \mathrm{e}^{-\mathrm{j} \omega_{s} t}
$$

where $E_{0}$ is the amplitude of the pulse, and $\omega_{s}$ is the angle frequency of the incident light.

Due to the difference of Rayleigh backscattering 
optical path, the returned lightwave field $E_{R}$ from each position can be expressed as follow:

$$
E_{R}=\hat{r}(z) E_{0} \mathrm{e}^{-\alpha z} \mathrm{e}^{\left(\mathrm{j} 2 \beta z-\omega_{s} t\right)}
$$

where $z$ is the spatial position of the returned lightwave, $\beta$ is the propagation constant, $\hat{r}(z)$ includes effects of the backscattering ratio and phase delay of Rayleigh backscattering at different spatial positions.

The interference model of $\Phi-O T D R$ is established and presented as shown in Fig. 1 [1].

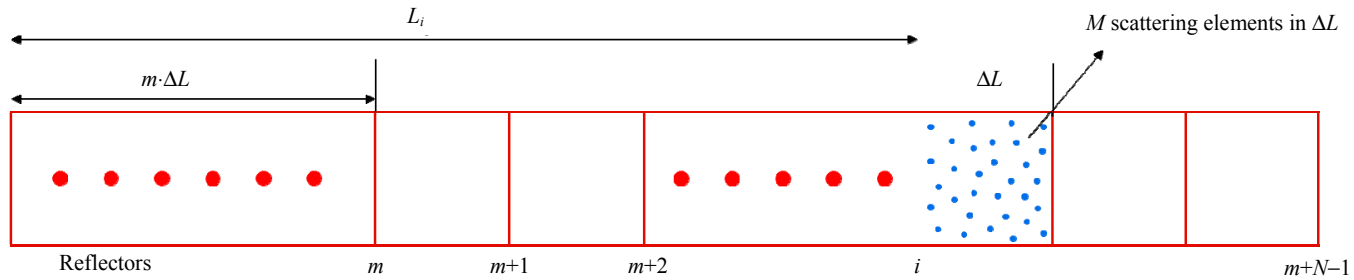

Fig. 1 Interference model of $\Phi$-OTDR [1].

First, the fiber is divided by the spatial resolution $\Delta L$ into $N$ segments. Each segment is regarded as a discrete reflector. Then, the received signal from each reflector is the superposition of a series of $M$ small Rayleigh backscattering centers within $\Delta L$. Therefore, disregarding the polarization of each scattering element, the parameter $\hat{r}(z)$ in (2) can be expressed as [1]

$$
\hat{r}=r_{i} \mathrm{e}^{\mathrm{j} \varphi_{i}}=\sum_{k=1}^{M} a_{k} \mathrm{e}^{\mathrm{j} \psi_{k}}
$$

where $r_{i}$ and $\varphi_{i}$ are amplitude and phase of the $i$-th fiber partition, respectively, and $a_{k}$ and $\psi_{k}$ are amplitude and phase of the $k$-th Rayleigh scattering center, respectively. $r_{i}, \varphi_{i}, a_{k}$, and $\psi_{k}$ are all random variables. $\psi_{k}$ is uniformly distributed from $-\pi$ to $\pi$, and $a_{k}$ and $\psi_{k}$ are independent. $r_{i}$ and $\varphi_{i}$ of each reflector have independent random distribution. Therefore, the spatial distribution of $r$ and $\varphi$ is Rayleigh distribution and uniform distribution, respectively, which can be expressed as [1]

$$
\begin{gathered}
p(r)= \begin{cases}\left(r / \sigma^{2}\right) \mathrm{e}^{-r^{2} / 2 \sigma^{2}}, & r>0 \\
0, & \text { otherwise }\end{cases} \\
p(\varphi)= \begin{cases}1 / 2 \pi, & -\pi<\varphi \leq \pi \\
0, & \text { otherwise }\end{cases}
\end{gathered}
$$

where $\sigma$ is the Rayleigh backscattering coefficient. Thus, $E_{R}$ can be expressed as

$$
E_{R}=r_{i} E_{0} \mathrm{e}^{-\alpha z} \mathrm{e}^{\mathrm{j}\left(2 \beta z+\phi_{i}-\omega_{s} t\right)}
$$

where $\alpha$ is the attenuation coefficient of the fiber. Then, the detected Rayleigh backscattering signal power corresponding to the $m$-th reflector $P_{m}$ is

$$
P_{m}=P_{0} \mathrm{e}^{-2 \alpha m \Delta L}\left|\sum_{i=m}^{m+N-1} r_{i} \mathrm{e}^{\mathrm{j} \varphi_{i}}\right|^{2} .
$$

Due to the integral effect, the detected Rayleigh backscattering signal power is the superposition result of the $W / \Delta L$ scattering centers within the spatial width $W$ corresponding to one pulse.

When Rayleigh backscattering lightwave propagates along the fiber with length of $L$, the phase delay $\varphi$ can be denoted as $\varphi=\beta L$. When the fiber is disturbed at position $z$, the fiber length, the core diameter and the core refractive index will change correspondingly, leading to phase delay change $\Delta \varphi$ of the Rayleigh backscattering light. The phase delay change $\Delta \varphi$ can be denoted as [2]

$$
\Delta \varphi=\beta \cdot \Delta L+L \cdot \Delta \beta=
$$

$$
\beta L \cdot \frac{\Delta L}{L}+L \cdot\left(\frac{\partial \beta}{\partial n}\right) \Delta n+L \cdot\left(\frac{\partial \beta}{\partial \alpha}\right) \Delta \alpha
$$

where $n$ and $\alpha$ are core refractive index and diameter, respectively. The three items in (8) represent phase changes introduced by the fiber deformation, photo-elastic effect, and Poisson effect, respectively. Therefore, by demodulating Rayleigh backscattering trace along the sensing fiber, external vibrations or acoustic waves can be recovered.

\section{Historical review of $\Phi$-OTDR}

As early as 1993, Henry F. Taylor et al. found that vibrations along optical fiber could be detected by demodulating coherent Rayleigh backscattering 
light intensity from a narrow linewidth laser [3]. Then, the demonstration of $\Phi-O T D R$ was published in 1994 [4], but the performance of $\Phi-O T D R$ at that time was quite poor due to the limitation of the light source performance. In 1998, J. Park et al. made a theoretical analysis for $\Phi-O T D R$ and demonstrated a $\Phi-O T D R$ based on a ring laser with linewidth of $<50 \mathrm{kHz}$, achieving $400 \mathrm{~m}$ spatial resolution and 3.3 signal to noise ratio (SNR) [1]. In contrast to conventional optical time domain reflectometry, $\Phi-O T D R$ has strict requirements on the frequency drift and linewidth of the light source. In 2003, a spectrally stable Er-fiber laser with low frequency drift rate $(<1 \mathrm{MHz} / \mathrm{min})$ by incorporating a long fiber passive feedback loop in the laser was developed [5].

The early application of $\Phi$-OTDR is to detect and locate intruders over long perimeters. Direct detection is applied in $\Phi-O T D R$ and the phase change induced by the intruders and anomalies can be indirectly detected by subtracting a $\Phi-O T D R$ trace from the trace undisturbed. In 2005, Ф-OTDR was firstly employed for intrusion detection [6]. Juan C. Juarez et al. demonstrated a $\Phi-O T D R$ based on a continuous-wave Er-fiber Fabry-Pérot laser with a narrow instantaneous linewidth $(\sim 3 \mathrm{kHz})$ as well as low frequency drift, achieving $12 \mathrm{~km}$ sensing distance and $100 \mathrm{~m}$ spatial resolution [7]. Later in 2007, they extended the sensing distance to $19 \mathrm{~km}$ with $200 \mathrm{~m}$ spatial resolution, and intruders on foot and vehicles near the cable line were consistently detected in real time in field tests [8]. Also, in 2007, the Fiber Optics Research Center (FORC) at University of Electronic Science and Technology of China (UESTC) initiated the research and development (R\&D) of $\Phi$-OTDR, which is the first group to study $\Phi-O T D R$ in China. Y. J. Rao et al. applied a high-power single-mode fiber laser (50 mW, NP Photonics, USA) with narrow linewidth $(\leqslant 3 \mathrm{kHz})$ to $\Phi$-OTDR, for the first time, in 2008 , achieving a sensing distance range of $14 \mathrm{~km}$ with an improved spatial resolution of $\sim 50 \mathrm{~m}$. This work paved a way for realization of practical $\Phi-O T D R$ systems [9]. In 2009, the authors proposed a distributed fiber-optic perturbation sensing system combined with $\Phi-O T D R$ and polarization-sensitive optical time domain reflectometry, which improved the accuracy of locating remote perturbations and reduced the noise with wavelet analysis effectively [10].

Not only the intensity but also the phase change of coherent Rayleigh backscattering light can be obtained via $\Phi-O T D R$. The intensity change is able to locate the disturbance position through direct detection, but it is merely suitable for the quantitative detection, instead of catching the changing waveform of the external vibration signal accurately. From the sensing mechanism of Ф-OTDR, it is known that outside environment change can cause the phase change, which means that the phase change of Rayleigh backscattering light can be quantitatively detected in real time, and that is the reason why the $\Phi-O T D R$ based on the phase demodulation algorithm, i.e., distributed acoustic sensing (DAS), has been widely concerned in recent years. In 2011, H. W. Cai et al. applied the digital coherent detection algorithm into $\Phi$-OTDR for the first time, and reconstructed the instantaneous electric field of Rayleigh backscattering light. As a result, the system obtained both amplitude and phase signal, with a sensing distance of $3.5 \mathrm{~km}$ and a spatial resolution of $5 \mathrm{~m}$ [11]. In 2013, the working mechanism of Ф-OTDR based on imbalanced Mach-Zehnder fiber interferometer and $3 \times 3$ coupler was systematically explained, to quantify multiple dynamic strain perturbations along $1 \mathrm{~km}$ fiber link, with $2 \mathrm{~m}$ spatial resolution and $500 \mathrm{~Hz}-5 \mathrm{kHz}$ response frequency [12]. In 2014, H. W. Cai et al. introduced frequency division multiplexing into a high sampling rate multi-pulse $\Phi-O T D R$, realizing a new system with $20 \mathrm{kHz}$ vibration detection bandwidth over $10 \mathrm{~km}$ sensing range [13].

For the past few years, researchers have further studied the phase demodulation methods for 
Ф-OTDR. A Ф-OTDR system with phase generated carrier technology based on imbalanced Michelson interferometer was proposed by Y. Shang et al. in 2015 [14]. The system with a spatial resolution of $10 \mathrm{~m}$ over $5 \mathrm{~km}$ sensing range achieved an acoustic phase sensitivity of $-151 \mathrm{~dB}$ re $\mathrm{rad} / \mu \mathrm{Pa}$ at $600 \mathrm{~Hz}$, and a minimum detectable acoustic pressure of $6 \mathrm{~Pa}$. In 2016, the authors made use of the technique based on in-phase and quadrature-phase (I/Q) demodulation and homodyne detection with a $90^{\circ}$ optical hybrid to implement a dynamic strain sensing system with a sensing range of $12.56 \mathrm{~km}$ and a spatial resolution of $10 \mathrm{~m}$ [15].

In some early works, due to the advantages of high spatial resolution, optical frequency domain reflectometry (OFDR) has been used for dynamic strain detection. Since chirped-pulse technique can break the tradeoff between spatial resolution and pulse width of $\Phi$-OTDR, several research groups developed distributed sensing system based on the chirp pulse method, essentially combining $\Phi-O T D R$ and OFDR technologies. In 2015, Z. Y. He et al. added a time gate into the traditional OFDR. Based on the time-gated digital optical frequency domain reflectometry (TGD-OFDR), the dynamic range and SNR were greatly enhanced, and the phase of optical signals was extracted over a long distance up to 29 $\mathrm{km}$ with $2.2 \mathrm{~m}$ spatial resolution [16]. In 2016, J. Pastor-Graells et al. utilized a $\Phi-O T D R$ with linearly chirped pulses and direct detection, as well as cross-correlation demodulation algorithm, and succeeded in achieving sensing distance of $1 \mathrm{~km}$ and the minimum detectable strain of $4 \mathrm{n} \varepsilon$ under the spatial resolution of $1 \mathrm{~m}$ [17]. In 2017, J. Pastor-Graells et al. successfully demonstrated a chirped-pulse Ф-OTDR (CP-ФOTDR) with a sensing distance of $75 \mathrm{~km}$ and a spatial resolution of $10 \mathrm{~m}$, by using bi-directional first-order Raman amplification, allowing to measure the smallest strain as low as $1 \mathrm{n} \varepsilon$ [18]. And then in 2019, H. F. Martins et al. highlighted the particular performance characteristics of CP-ФOTDR, pointing out that it will be a solid, highly competitive, and extraordinarily simple method for distributed fiber sensing [19].

The merits of combining $\Phi-O T D R$ and OFDR technologies are more than just that, as the researchers keep pushing the sensitivity limit of Ф-OTDR. In 2018, a TGD-OFDR based on optical intensity modulator was proposed by $\mathrm{D}$. Chen et al., achieving a strain resolution of $245.6 \mathrm{p} \varepsilon / \sqrt{ } \mathrm{Hz}$ and a spatial resolution of $0.8 \mathrm{~m}$ along $9.8 \mathrm{~km}$ sensing fiber in the case of fading suppression [20]. In 2020, the authors reported the use of unidirectional first-order Raman amplification on the DAS system with a sensing distance of $103 \mathrm{~km}$, of which the strain resolution was $97 \mathrm{p} \varepsilon / \sqrt{\mathrm{Hz}}$ with $9.3 \mathrm{~m}$ spatial resolution [21].

After years of development, $\Phi-O T D R$ is no longer confined to the laboratory research. Recently, the related researchers are taking initiative in employing $\Phi-O T D R$ in geological exploration, security monitoring and other fields. In 2018, Nature Communications reported the study of DAS with $4 \mathrm{~m}$ spatial resolution along $15 \mathrm{~km}$ sensing range, recording the seismic signals from natural and man-made sources, further allowing to infer new dynamic fault processes [22]. Then in 2019, Ethan F. Williams et al. took advantage of $41.5 \mathrm{~km}$ undersea optical cable to extract critical waveforms through the DAS array for undersea seismic monitoring, which showed a significant potential for DAS in next-generation submarine seismic networks [23].

\section{Long-distance $\Phi-O T D R$}

For practical applications, how to extend sensing range while maintaining a relatively high spatial resolution becomes a challenge in $\Phi-O T D R$. Due to weak Rayleigh backscattering light and exponentially decreased sensing signal along optical fiber, long-distance $\Phi-O T D R$ is quite difficult to realize. In traditional $\Phi$-OTDR, intensity of Rayleigh backscattering signal is increased by enhancing injected pulse power using erbium-doped fiber amplifier (EDFA). However, the input power is 
confined by the influence of stimulated Brillouin scattering (SBS) [24]. Under this restriction, the sensing length of $\Phi$-OTDR only achieves $25 \mathrm{~km}$ (35 $\mathrm{m}$ spatial resolution) [5]. In order to break through the limitation, Y. J. Rao et al. used the distributed amplification method to extend the Ф-OTDR sensing range to $62 \mathrm{~km}$ (100 m spatial resolution) with bi-directional $1^{\text {st }}$-order distributed Raman amplification (DRA), for the first time, in 2009 [25], which paved the way to realize longdistance $\Phi-O T D R$ by using distributed optical amplification technology. Afterwards the forward $1^{\text {st }}$-order DRA and heterodyne detection were applied to $\Phi-O T D R$, and the sensing distance has been extended to $103 \mathrm{~km}$ ( $15 \mathrm{~m}$ spatial resolution) [26]. Furthermore, H. F. Martins et al. improved
SNR of incident pulse light by using semiconductor optical amplifier and optical switch with bi-directional $1^{\text {st }}$-order DRA, extending sensing distance to $125 \mathrm{~km}$ (10 m spatial resolution) by reducing the influence of relative intensity noise (RIN) transfer from Raman pump (RP) with balanced photodetector in 2014 [27]. Subsequently, the $2^{\text {nd }}$-order DRA was applied to realize $131 \mathrm{~km}$-long $\Phi-O T D R$ [24]. In 2014, the authors proposed a hybrid distributed amplification (HDA) method and achieved a sensing distance of $175 \mathrm{~km}$ in $\Phi-O T D R$ system, for the first time [28], greatly increasing the record repeaterless sensing distance of $\Phi-O T D R$ by $\sim 50 \mathrm{~km}$. Figure 2 summarizes the sensing distance improvement of $\Phi$-OTDR in recent years $[5,24,25,27-29]$.

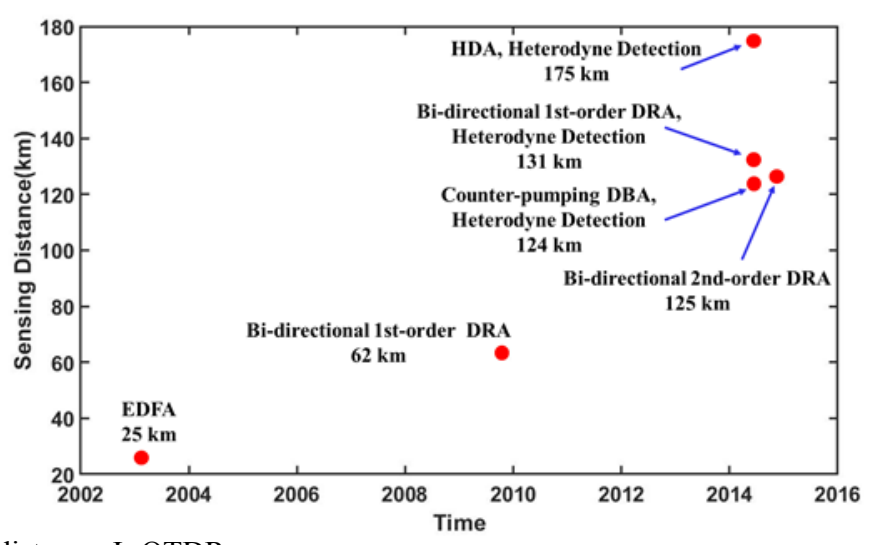

Fig. 2 Development of long-distance $Ф$-OTDR.

\section{1 Ф-OTDR with bi-directional DRA}

Since the emergence of relatively mature Raman pumped laser in 1990s, the DRA technology has been widely used in optical fiber communication and sensing systems, which plays a key role in sensing distance extension due to its lower noise figure and flatter gain profile compared with EDFA [30-33].

As the power distribution of Rayleigh backscattering and the limitation of SBS on pump power need to be considered simultaneously, and also the Brillouin backscattering light cannot be separated from Rayleigh backscattering light in direct detection, the entering power of pulse signal and RP into the fiber is limited. A combined technology scheme based on the bi-directional $1^{\text {st }}$-order DRA and heterodyne detection is proposed by the authors. This scheme achieves the overall improvement of the $\Phi$-OTDR sensing distance, spatial resolution and sensitivity with sensing distance $>100 \mathrm{~km}$ and spatial resolution $<10 \mathrm{~m}$. The system diagram is shown in Fig. 3 [24].

Limited by the long trigger period, the maximum response frequency of long-distance $\Phi$-OTDR is much lower than that of short distance $\Phi-O T D R$. The system works under the optimized power combination as shown in Fig. 4(d). The position with the lowest SNR is located near $100 \mathrm{~km}$. Therefore, $97.51 \mathrm{~km}$ is selected as the test location in the vibration and intrusion experiment. In order to 
verify the maximum response frequency of the system, $5 \mathrm{~m}$ optical fiber wound on a cylindrical piezoelectric (PZT) at $97.51 \mathrm{~km}$ was used for vibration testing. The total electrostriction displacement is $\sim 3 \mu \mathrm{m}$ when the driven voltage is $3 \mathrm{~V}$. The driven signal applied to PZT cylinder is a sinusoidal wave with $3 \mathrm{~V}$ peak-peak voltage and
$25 \mathrm{~Hz}$ to $375 \mathrm{~Hz}$ frequencies. The auto-power spectrums of $97.51 \mathrm{~km}$ when vibrations with different applied frequencies are shown in Fig. 5. The peak frequency of each spectrum is in good agreement with the vibration frequency and has high SNR, which shows that this system has good ability for high frequency response.

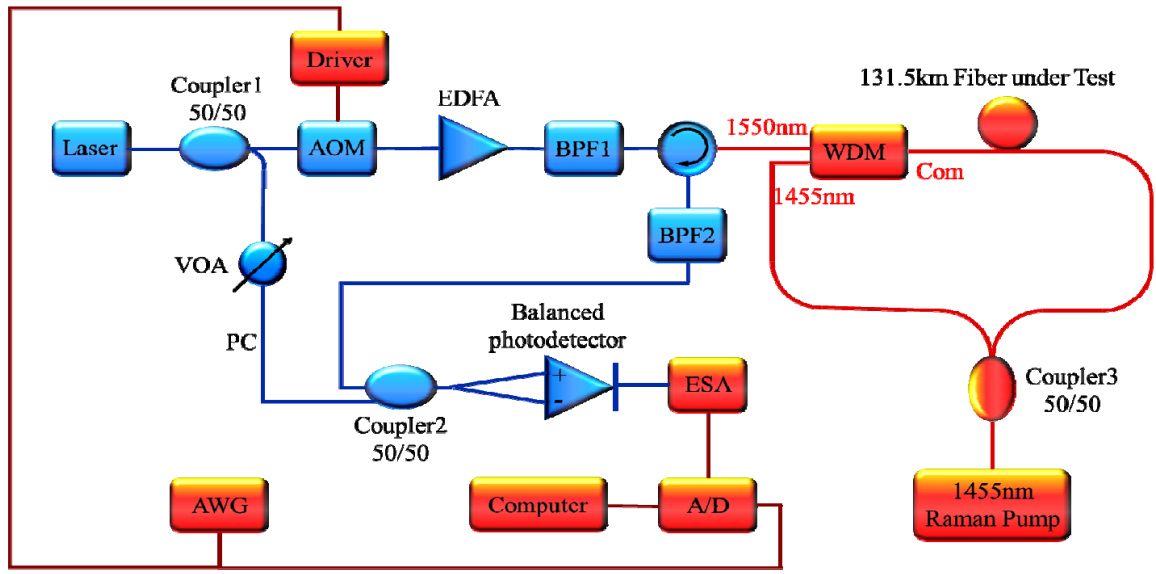

Fig. 3 Ф-OTDR system based on bi-directional $1^{\text {st }}$-order DRA and heterodyne detection.

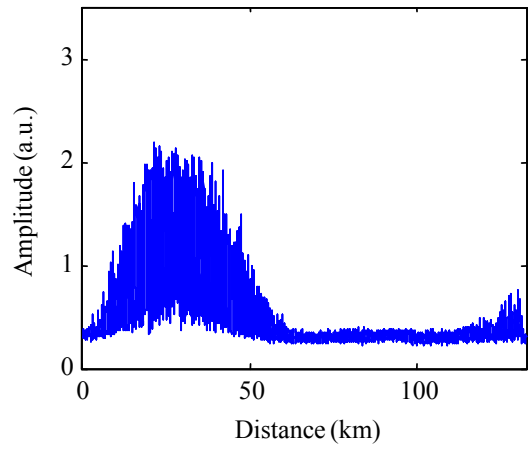

(a) $P_{P}=-36.3 \mathrm{dBm}$ and $P_{R}=32.7 \mathrm{dBm}$

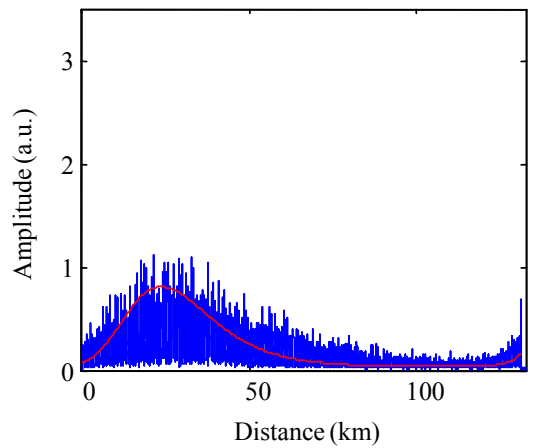

(c) $P_{P}=-36.3 \mathrm{dBm}$ and $P_{R}=30.6 \mathrm{dBm}$

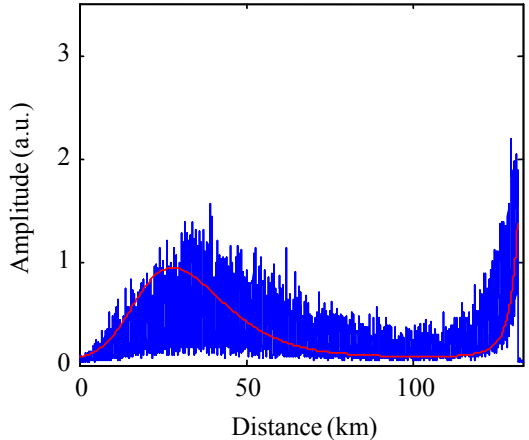

(b) $P_{P}=-41.7 \mathrm{dBm}$ and $P_{R}=31.5 \mathrm{dBm}$

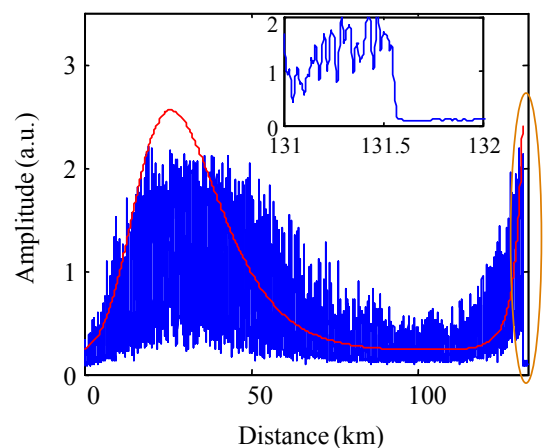

(d) $P_{P}=-36.3 \mathrm{dBm}$ and $P_{R}=31.5 \mathrm{dBm}$

Fig. 4 Rayleigh backscattering signals with different combinations of probe and pump light, where the curves in (b), (c), and (d) are the results of simulation. 


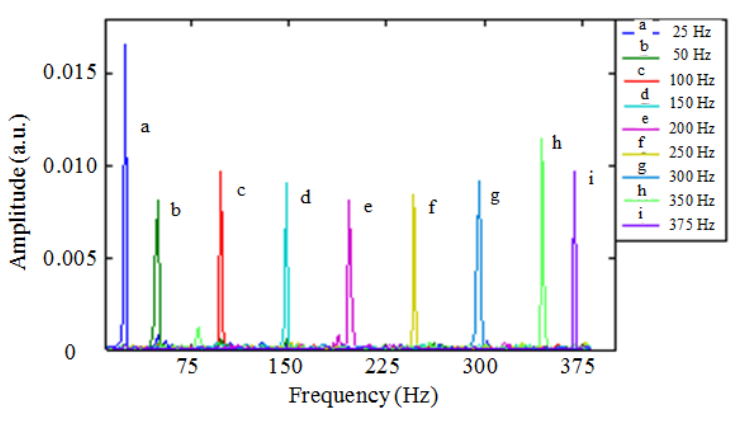

Fig. 5 Auto-power spectrum in different vibration frequencies at the location of $97.51 \mathrm{~km}$.

\subsection{Ultra-long $\Phi-O T D R$ with HDA}

Distributed Brillouin amplification (DBA) technology based on SBS is another distributed amplification technology. Since the gain coefficient of SBS is much higher than that of stimulated Raman scattering (three orders of magnitude), the pump power requirement for DBA is much lower than that of the DRA to achieve the same amplification effect. In 2014, we introduced the DBA technology into the optical fiber sensing system for the first time, and achieved a $\Phi-O T D R$ with $100 \mathrm{~km}$ sensing distance and $10 \mathrm{~m}$ spatial resolution using $6.4 \mathrm{dBm}$ counter-pumping DBA. Moreover, the SNR of the signal obtained is higher than that of using $28.0 \mathrm{dBm}$ counter-pumping DRA [34].

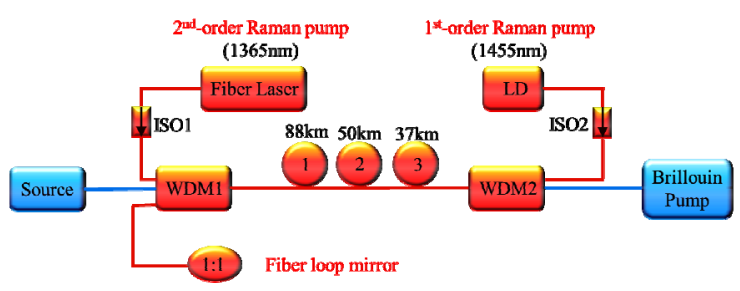

Fig. 6 Principle diagram of the HDA method.

In order to extend the sensing distance of $\Phi-O T D R$ as long as possible, a novel HDA method shown in Fig. 6 was proposed, which utilized the combination of three different pumping schemes to push the sensing range of $\Phi$-OTDR to the world record of $175 \mathrm{~km}$ ( $25 \mathrm{~m}$ spatial resolution and $\sim 70 \mathrm{~dB}$ roundtrip fiber-loss). Based on different Brillouin frequency shifts (BFS) of the fiber, the sensing fiber used can be divided into three sections. The BFS of Segment 2 differs greatly from that of Segments 1 and 3.

The co-pumping $2^{\text {nd }}$-order Raman amplification based on random fiber lasing provides gain mainly in the first half of the whole span (Segment 1), and then the counter-propagating Brillouin pump (BP) starts to dominate the amplification in Segment 2; at last, the $1^{\text {st }}$-order Raman amplification takes over the signal amplification in the last $37 \mathrm{~km}$ of the fiber (Segment 3). Each pump amplification takes effect in each fiber segment, achieving the distributed amplification over the entire fiber transmission distance. As a comparison of HDA scheme, the system with ultra-long Raman fiber laser (URFL)-based $2^{\text {nd }}$-order RP is also analyzed. The pump power simulation results are shown in Fig. 7. It can be seen that, different from the URFL scheme, the intensity of BP firstly increases from $175 \mathrm{~km}$ until reaching its maximum at around $155 \mathrm{~km}$, and then slowly attenuates, which manifests the amplification effect of the $1455 \mathrm{~nm} 1^{\text {st }}$-order RP injected at $175 \mathrm{~km}$.

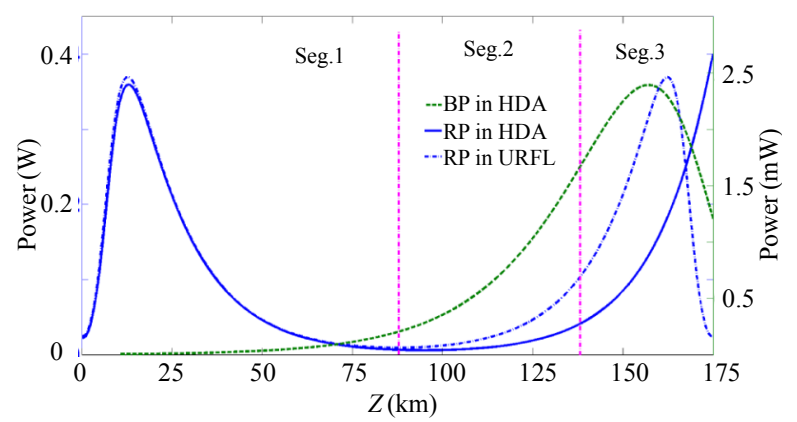

Fig. 7 Power distribution of $1455 \mathrm{~nm} \mathrm{RP}$ and $1550 \mathrm{~nm}$ BP.

Figure 8 shows the Rayleigh backscattering traces of the probe pulse in simulation with two different amplification schemes. In Fig. 8, with URFL scheme, Rayleigh backscattering trace reaches it maximum at around $37 \mathrm{~km}$ and then continuously decreases as the Raman gain cannot completely compensate the fiber loss during transmission. Although the effects of the counter-propagating $1^{\text {st }}$-order RP are implemented in 
the first half of the fiber, the intensity of Rayleigh backscattering trace still drops to a quite low level. In contrast, with the HDA scheme, the signal intensity is improved in Segment 2 with the aid of BP and further amplified in Segment 3 by the counter-propagating $1^{\text {st }}$-order RP. The simulation results in Fig. 8 suggest that the HDA scheme can be used to achieve an ultra-long-distance $\Phi$-OTDR system.

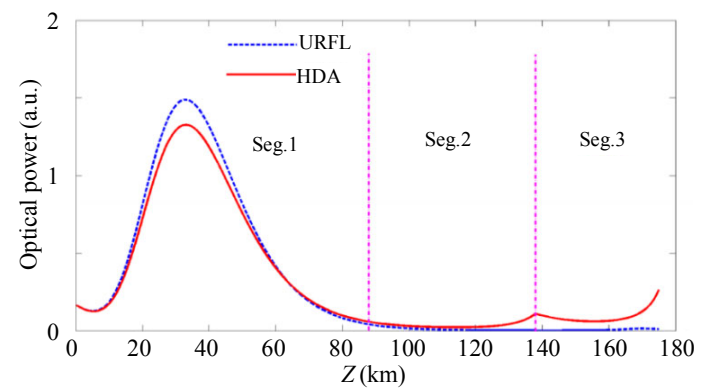

Fig. 8 Rayleigh backscattering trace comparison between URFL and HDA schemes in simulation.
As a verification of the HDA scheme, a $\Phi-O T D R$ with $175 \mathrm{~km}$ sensing range is set up based on heterodyne detection technology, as shown in Fig. 9 [28]. Based on the above experimental setup, the performances of $\Phi$-OTDR with different pump conditions are tested. In the HDA scheme, the frequency shift of the BP to the probe signal is at first set to be $11.034 \mathrm{GHz}$ in order to maximize SBS gain in Segment 2. The detected interference traces (averaged over 32 times, corresponding to $7.8 \mathrm{~Hz}$ bandwidth) are shown in Fig. 10. Compared with the case without the BP, it is explicitly shown that the input BP has significantly improved the signal intensity. Meanwhile, without the assistance of the $1^{\text {st }}$-order RP, the BP has almost no effect on Segment 2, as shown in Fig. 10(b), for the BP is too weak to provide gain for the probe pulse.

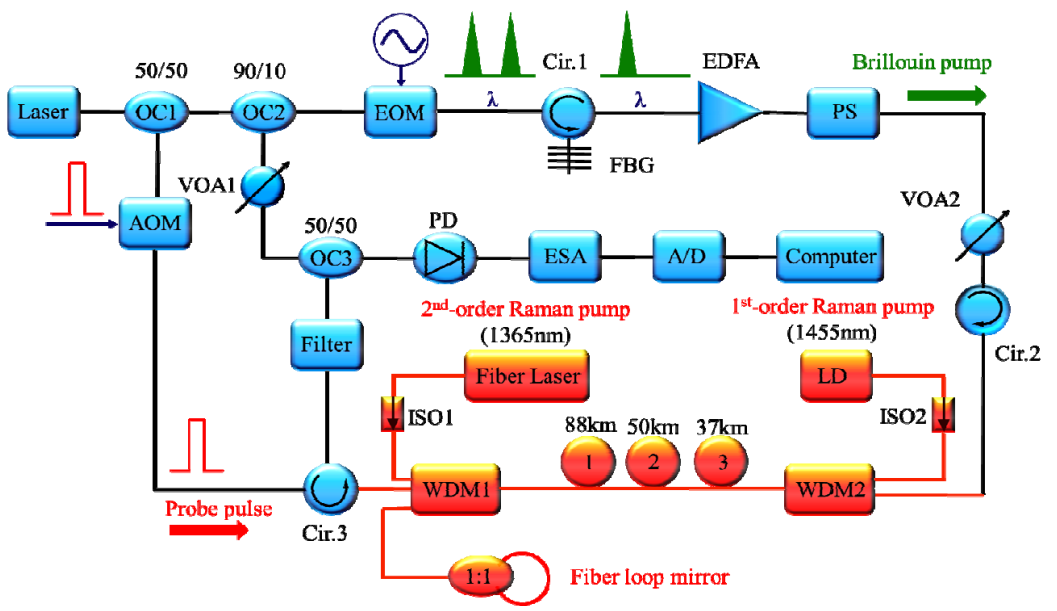

Fig. 9 Experimental setup of $\Phi-O T D R$ based on the HDA method.

The Brillouin gain spectrum of the fiber is related to the external temperature, which will affect the amplification performance of the SBS. In the experiment, the BP is set at different frequencies to test the sensitivity of the system to the ambient temperature. The experimental results are shown in Fig. 11. Figure 11(a) shows the $\Phi-O T D R$ traces without the BP and with the BP frequency shift set at $11.022 \mathrm{GHz}$, while Fig. 11(b) shows the $\Phi$-OTDR traces without the $\mathrm{BP}$ and with the $\mathrm{BP}$ frequency shift set at $11.047 \mathrm{GHz}$. It can be observed that the frequency of the BP does affect the performance of the amplification, to a certain extent. However, compared with the case without the BP, the enhancement of the signal quality is notable even the BP frequency varies from $11.022 \mathrm{GHz}$ to $11.047 \mathrm{GHz}$ (the frequency is $25 \mathrm{MHz}$ corresponding to $25{ }^{\circ} \mathrm{C}$ environmental temperature variation). As a result, $\Phi$-OTDR based on the HDA technology shows good adaptability to the temperature change (the BFS variation of the fiber). 


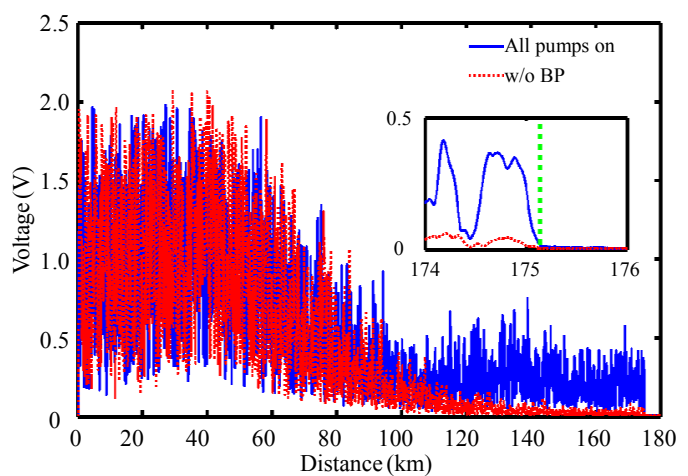

(a)

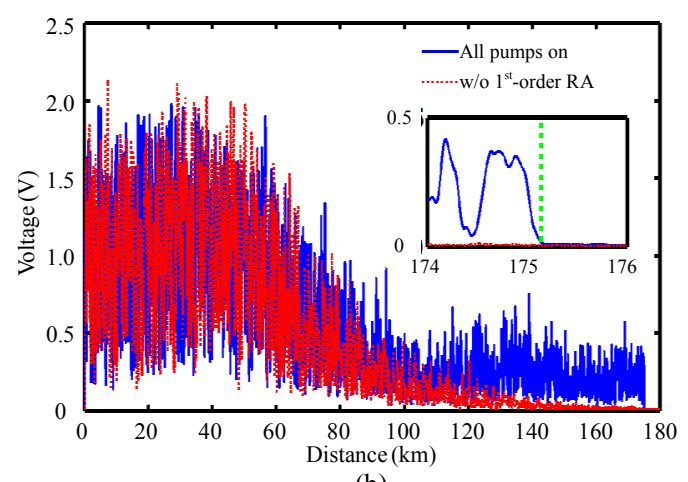

(b)

Fig. 10 Ф-OTDR traces: (a) all pumps on vs BP off and (b) all pumps on vs $1455 \mathrm{~nm}$ semiconductor RP off.

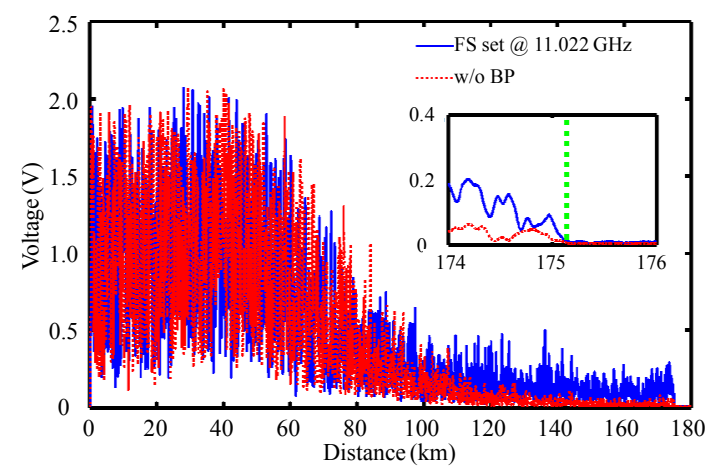

(a)

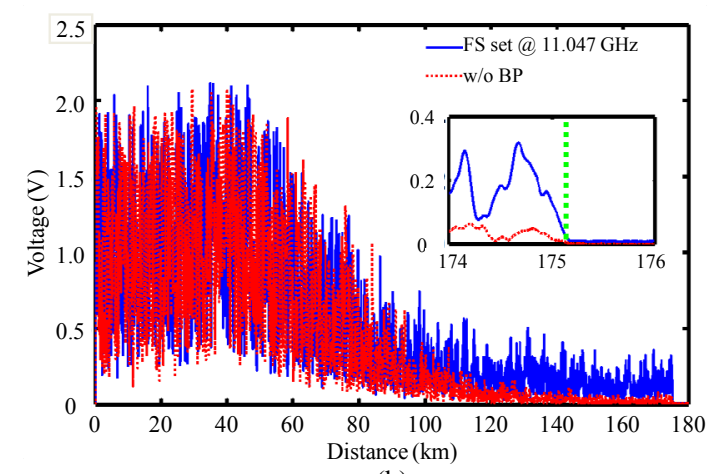

(b)

Fig. 11 Ф-OTDR traces: (a) BP frequency shift at $11.022 \mathrm{GHz}$ vs without BP; (b) BP frequency shift at $11.047 \mathrm{GHz}$ vs without BP. Insets: comparison of the signal inside the fiber and the noise outside the fiber.
Three monitoring points located at $87.3 \mathrm{~km}$, $136.6 \mathrm{~km}$, and $174.7 \mathrm{~km}$ are tested, respectively, with wavelet denoising method employed to extract effective perturbation information, which can improve the sensing performance of the $\Phi-O T D R$. The results of perturbation detection are shown in Fig. 12. When perturbation is applied at the $174.7 \mathrm{~km}$ monitoring point, the achieved SNR of the demodulated intrusion signals can be as high as $12.1 \mathrm{~dB}$. Figure 12(b) shows the multiple-point monitoring ability of the system, and perturbations at $87.3 \mathrm{~km}$ and $136.6 \mathrm{~km}$ are detected simultaneously, with $11.6 \mathrm{~dB}$ and $12.6 \mathrm{~dB}$ SNR, respectively. This hybrid amplification scheme can be further optimized to support even longer sensing range, and it can be extended to other fiber sensing systems.

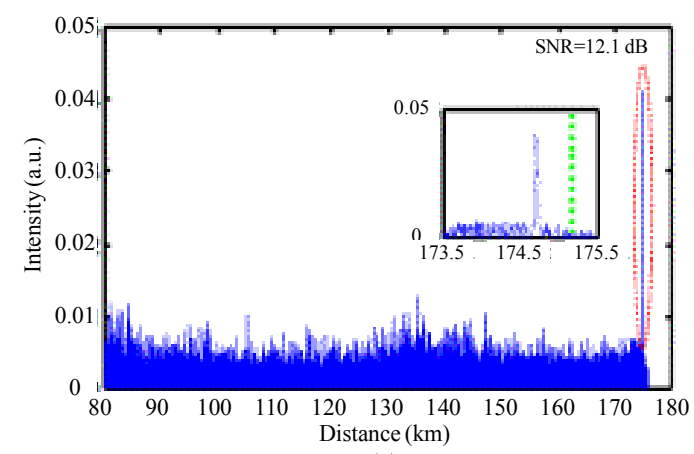

(a)

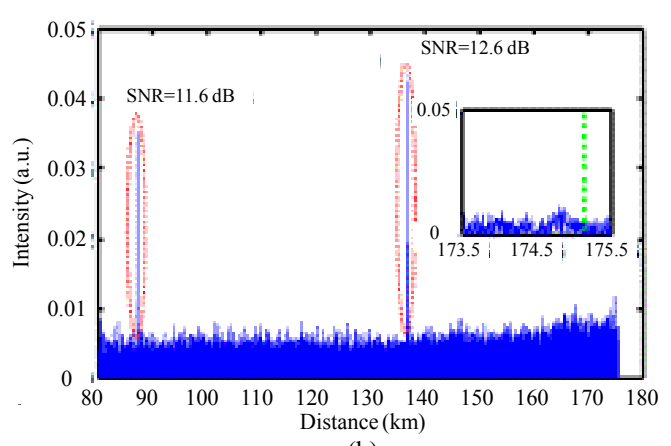

(b)

Fig. 12 Demodulated intrusion signal at different locations: (a) single-point perturbation at $174.7 \mathrm{~km}$ and (b) two-point perturbation at $87.3 \mathrm{~km}$ and $136.6 \mathrm{~km}$. Insets: comparison of the signal inside the fiber and the noise outside the fiber.

\section{4. Ф-OTDR with coherent detection}

Coherent detection, which is an indispensable technique in modern telecommunication, has been 
proved to be an effective approach to boost the performance of intensity demodulation $\Phi$-OTDR $[28,34]$; on the other hand, it can be naturally adopted to DAS [15, 35, 36]. Compared with other phase demodulation schemes, such as receiving interferometer [37], dual-pulse [38], and correlation with chirped pulse $[17,18]$, coherent detection has its unique advantages due to its extremely high scalability.

In terms of intensity demodulation $\Phi$-OTDR, $\Phi-O T D R$ utilizing coherent detection provides the significant gain from local oscillator signal, which is essentially beneficial for long-distance sensing. In terms of DAS, the coherent detection $\Phi-O T D R$ has become one of the main solutions. The DAS system based on coherent detection $\Phi-O T D R$, as shown in Fig. 13, has achieved $100 \mathrm{~km}$ sensing range with $8.2 \mathrm{~m}$ spatial resolution [39]. Its advantages lie not only in sensing range, but also in homodyne detection, vector demodulation, measurement bandwidth enhancement, fading elimination, etc.

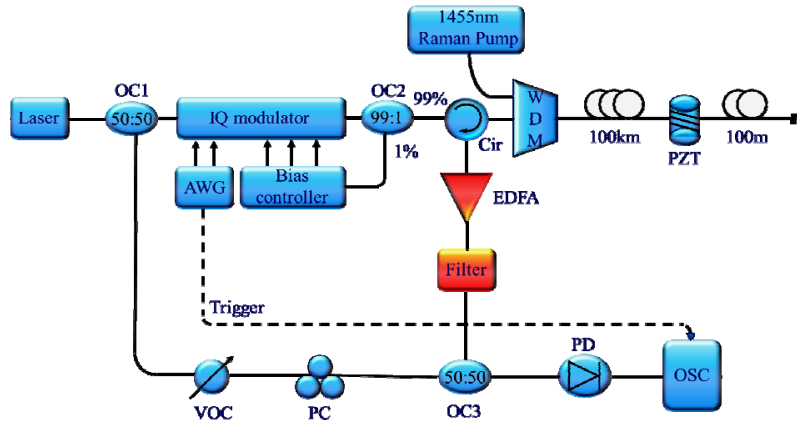

Fig. 13 Experimental setup of $100 \mathrm{~km}$ DAS system based on coherent detection [39].

The homodyne detection can be realized in coherent detection $\Phi$-OTDR by using a $90^{\circ}$ optical hybrid in Fig. 14 [15]. The Rayleigh backscattering signals detected by homodyne schemes is located in baseband, therefore only low-speed analog digital converter (ADC) is sufficient, which is beneficial for real-time data processing and storage. Besides, assisted with I/Q demodulation, the phase information of the light signal can be demodulated without an interferometer structure.

The lightwave vector demodulation based on coherent detection $\Phi-O T D R$ can acquire more information from Rayleigh backscattering field.
Combined with the $90^{\circ}$ optical hybrid, the polarization state can be distributedly measured [40, 41]. After that, the experiment was conducted and successfully demodulated the birefringence information in a $2 \mathrm{~km}$ spun SSMF with $8.6 \mathrm{~cm}$ spatial resolution (as shown in Fig. 15), which could play a significant role in high-sensitivity bending, stressing, and twisting sensing [42]. However, $90^{\circ}$ optical hybrid has some inherent imperfections, which will cause distortions in demodulated sensing signal [43]. According to the characteristics of $\Phi-O T D R$ received signals, the amplitude imbalance and phase offset of the hybrid can be real-time compensated [44].

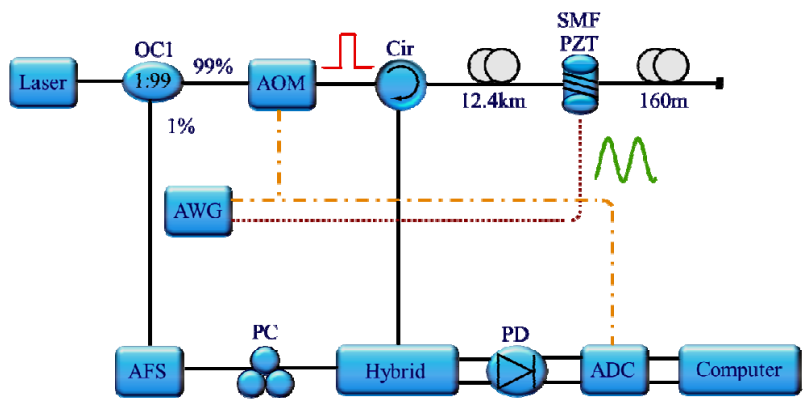

Fig. 14 Experimental setup of coherent detection $\Phi-O T D R$ based on homodyne detection [15]. 


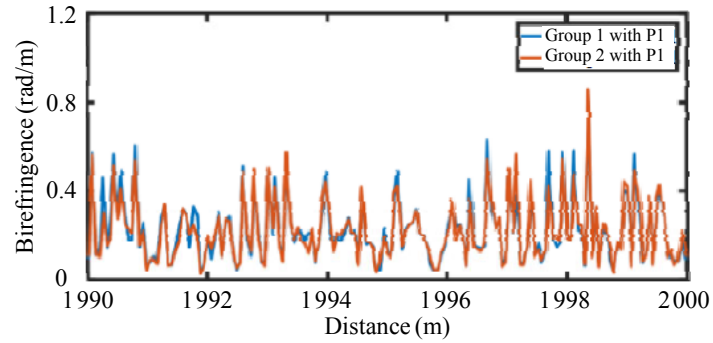

(a)

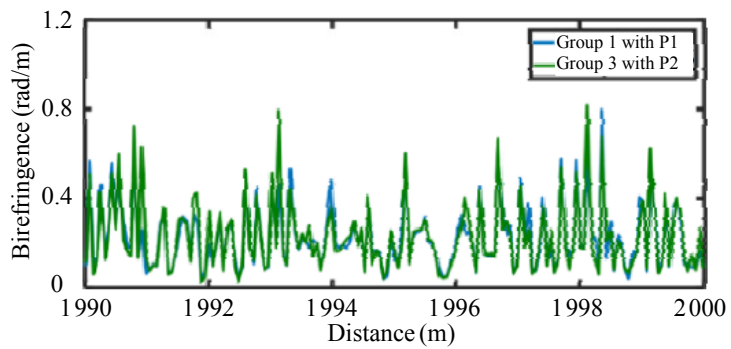

(b)

Fig. 15 Measurement birefringence results through coherent detection $\Phi$-OTDR [42].

The measurement bandwidth of coherent detection $\Phi-O T D R$ can be improved by various methods. For example, utilizing positive and negative beat frequencies, the measurement bandwidth is doubled in DAS system [45, 46]; based on interleaved chirped pulses, the measurement bandwidth is increased by multiple times in quasi-distributed acoustic sensing system and the system schematic is shown in Fig. 16 [47]. It should be noted that the probe pulses of aforementioned methods are all generated by I/Q modulator. However, the transfer function of the $\mathrm{I} / \mathrm{Q}$ modulator is non-linear, which makes the quality of the modulated optical signal not optimal. In order to solve this problem, the pre-distortion method is carried out to generate high-quality chirped pulses and improve the SNR of the system [48].

Fading phenomenon occurs in most $\Phi$-OTDR systems, which will reduce the sensor's performance, but it can be well suppressed by the coherent detection scheme. The number of fading points is proportional to the SNR of Rayleigh backscattering signals. Using Golay pulse coding, the SNR of coherent detection $\Phi-O T D R$ can be enhanced by $\sqrt{N} / 2$ times [49], and it can be further improved after using bipolar coding [50]. In addition, Kramers-Kronig receivers can suppress the noise caused by the signal-signal beat interference and improve the signal quality [51]. However, these methods to improve the SNR have an upper limit. A common way for further fading elimination is to aggregate $K$ statistically independent channels. These channels can be obtained simply by spectrum extraction and remix method, only a single rectangular probe pulse is required [52]. For the selection of aggregate channel numbers, through theoretical calculations and experimental verification, the fading suppression shows rather efficient in the case of 4 to 10 aggregated channels [53]. Furthermore, based on the coherent detection, coherent optical time domain reflectometry (COTDR) is inherently immune to interference fading. Assisted with sub-chirped-pulse extraction algorithm (shown in Fig. 17), the external disturbance can be extracted by simply using a single chirped-pulse [54], which is several orders of magnitude faster than traditional COTDR.

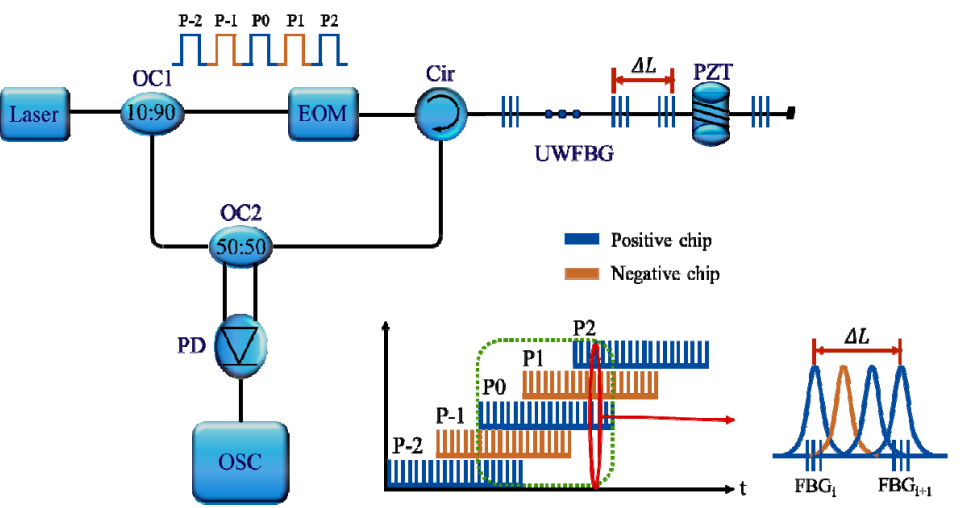

Fig. 16 Multiple times measurement bandwidth improvement through coherent detection $\Phi-O T D R$ [47]. 


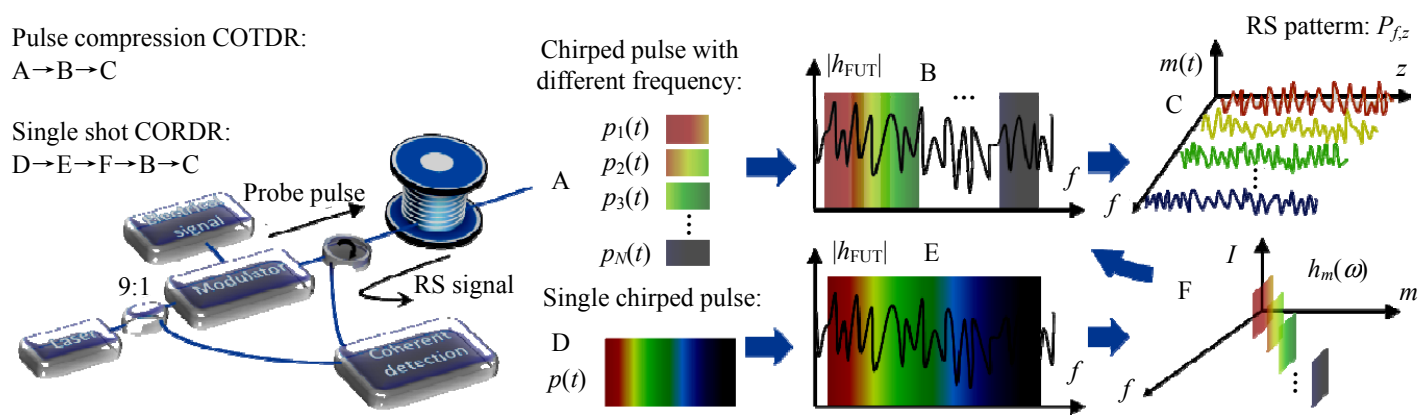

Fig. 17 Principle of sub-chirped-pulse extraction algorithm based on coherent detection [54].

\section{Applications of $\Phi$-OTDR}

\subsection{Train positioning and speed measuring with Ф-OTDR}

Currently, railway operation is based on the Communication Based Train Control (CBTC) to ensure the traffic safety of trains, and the CBTC needs to obtain the information of both real-time position and speed of trains [55]. Although the track circuit has been taken lightning protection measures, in some extreme weather conditions, such as strong lightning, it still has the risk to be damaged and may fail to work, leading to train operation safety accidents [56]. Therefore, researches on new train positioning and speed measurement systems that can resist electromagnetic interference are of great significance in the field of rail transit.

We show that by connecting the communication optical fiber buried under or near the rails, Ф-OTDR can determine the position of the train by locating the vibration source in real-time, for the first time, in 2014. Also, the speed of the train can be detected through differential of real-time results of positioning [57]. As $\Phi-O T D R$ has the characteristics of long sensing distance, real-time response, and immunity to electromagnetic interference, it makes positioning and speed monitoring of high-speed train, based on vibration detection, feasible.

The $\Phi-O T D R$ host of the monitoring center is connected to a 12.1-km-long optical cable below the rail side. The schematic diagram of the $\Phi$-OTDR is shown in Fig. 18 [57]. A narrow linewidth semiconductor laser has a very small frequency drift and is modulated by an acoustic-optic modulator (AOM) with pulse repetition rate of $1 \mathrm{kHz}$ and pulse width of $200 \mathrm{~ns}$, corresponding to a spatial resolution of $20 \mathrm{~m}$. Utilizing an ADC with $50 \mathrm{MSamples} / \mathrm{s}$, the signals are processed and analyzed by a computer in real time.

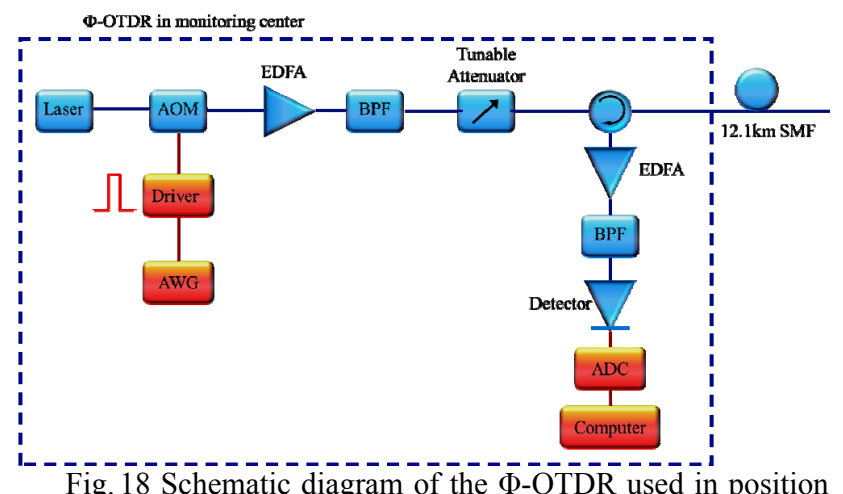

Fig. 18 Schematic diagram of the $\Phi$-OTDR used in position and speed monitoring of trains.

The relative positions among the optical cable, railways, and trains are shown in Fig. 19. The $12.1 \mathrm{~km}$ cable buried in soil with a depth range from $0.7 \mathrm{~m}-1.5 \mathrm{~m}$ is almost parallel to two railways with a separation distance of $15 \mathrm{~m}-20 \mathrm{~m}$ away from the nearest railway. The 10.2-km-long cable is within the monitoring zone. Two trains ran in opposite on two parallel tracks with comparatively steady speed. Vibration generated by running trains is transmitted to the cable through the soil and strain is applied on the optical cable. The $\Phi-O T D R$ in the monitoring center continuously detects the vibration of the whole optical cable link.

Since the direct detection scheme is adopted, more averaging times are required. The original Rayleigh backscattering curve is first averaged 25 times to reduce the thermal noise influence 
generated by the PD, thereby improving the detection SNR. When two trains pass by at $5.53 \mathrm{~km}$, the detected original vibration signal is shown in Fig. 20(a), and the vibration signal after wavelet denoising is shown in Fig. 20(b). The SNR of the vibration signal after denoising is significantly improved, and it can be seen that when the train approaches-passes-away from the certain position, the signal grows from nothing, gradually becomes stronger, and finally decays. After wavelet denoising, the vibration signal intensity at all positions in each period can be quantified by root mean square (RMS). Taking $70 \mathrm{~s}$ and $378 \mathrm{~s}$ as examples, the vibration levels at all positions are shown in Fig. 21. The length of the space between the arrows represents the range of vibration affected by the trains, which is $1100 \mathrm{~m}$ and $960 \mathrm{~m}$, respectively, and is slightly longer than the length of the trains, so the length of the two trains can be estimated according to the results.

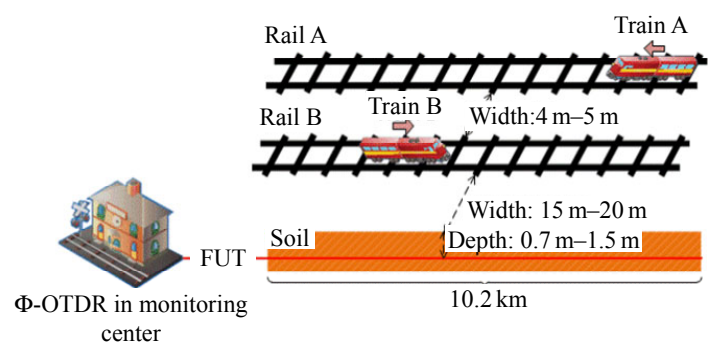

Fig. 19 Relative positions between the optical cable, trains, and railways.

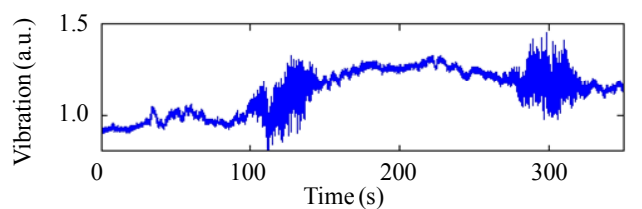

(a)

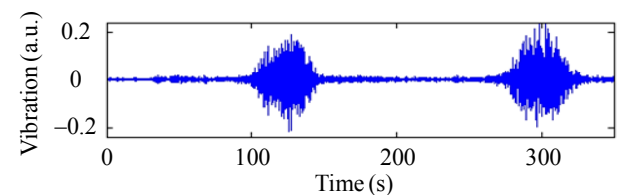

(b)

Fig. 20 (a) Original time series of vibrations and (b) vibration signals after wavelet denoising.

The vibration level-time-space intensity diagram obtained during the whole train operation is shown in Fig. 22(a). Through RMS, sliding variance, and peak detection method, the rising and falling edges of the vibration corresponding to the beginning and end of the train are obtained, as shown in Fig. 22(a). Finally, the displacement curve of the train is derived to obtain the speed curve of the train, as shown in Fig. 22(b). From Fig. 22(b), when Train A is running left at $10 \mathrm{~km}$ in $115 \mathrm{~km} /$ hour, Train B has not entered the monitoring area yet, which enters the monitoring area at $174 \mathrm{~s}$ in $105 \mathrm{~km} /$ hour. At $194 \mathrm{~s}$, the two trains converge at $3.9 \mathrm{~km}$, vibrating over a very wide area due to resonance, as shown in the crossing area in Fig.22(a). The two trains leave the monitoring area at $420 \mathrm{~s}$ and $255 \mathrm{~s}$, respectively, and the entire fiber tends to be quiet.

The experiments are sufficient to illustrate the feasibility and future potential of $\Phi$-OTDR in this field: $\Phi-O T D R$ can be used as an effective supplement and alternative to the current CBTC, and its measurement results are more valid than those of the current track circuit in extreme lightning weather. Therefore, $\Phi-O T D R$ is expected to play an important role in the future for structural health and safety monitoring in rail transit.

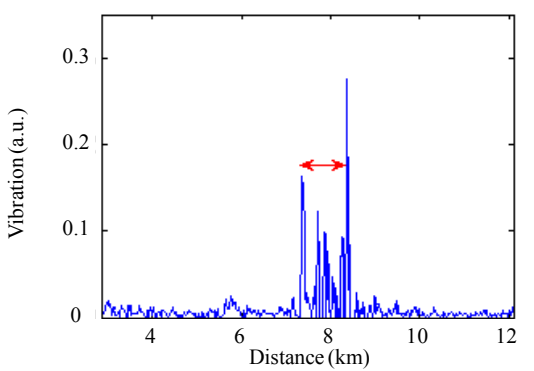

(a) Vibration generated by Train A at $70 \mathrm{~s}$

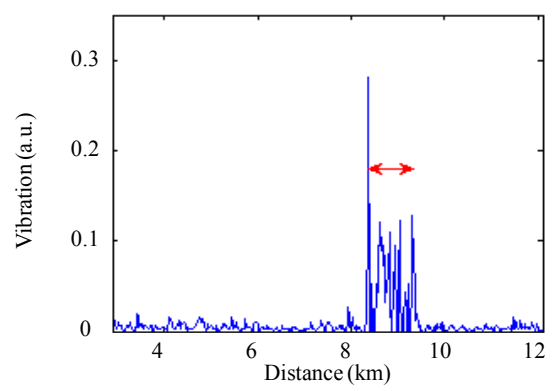

(b) Vibration generated by Train B at $378 \mathrm{~s}$

Fig. 21 Vibration signals of trains A and B at two different time points. 


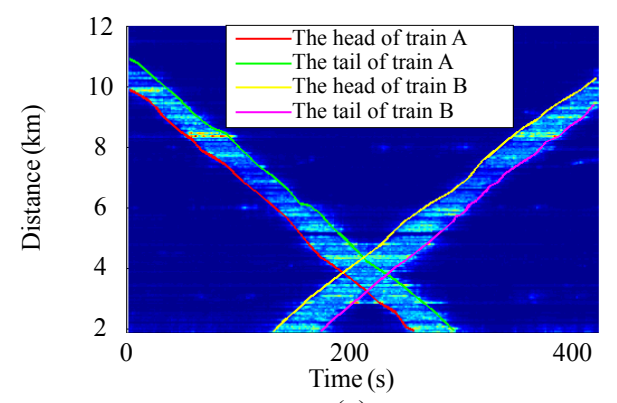

(a)

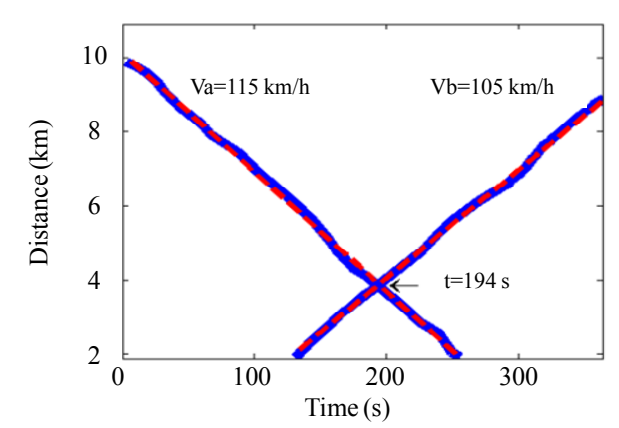

(b)

Fig. 22 (a) Vibration intensity vs space \& time and the edges of every location period and (b) the two edges of the two trains' heads (blue lines). The red lines are the fitting traces of the two trains.

\subsection{Oil pipeline safety monitoring with DAS based on $\Phi$-OTDR}

DAS based on phase-demodulated $\Phi$-OTDR shows the ability to detect changes of the external vibration and sound waves, including acoustic amplitude, frequency, and phase information. In the oil industry, DAS has shown unprecedented application prospects. The application aspects of DAS are mainly divided into two categories: seismic wave detection and oil pipeline safety monitoring, covering the exploration, production and transportation of the oil industry, which are of great economic and social significance [58].

The majority of pipeline accidents are caused by third party interference (TPI), which leads to not only the loss of petroleum products, but more importantly, environmental pollution and serious ecological disaster [58]. The processes of TPI will generate seismic waves in the pipeline. Thus, DAS can be connected with the communication cable of the booster station to complete long-distance intrusion detection. The situation of oil pipelines on site is relatively complex, most areas are close to roads and railways, and the vibration of vehicles will cause a lot of interference to the monitoring system. Therefore, it is necessary to recognize all kinds of vibration signals to distinguish different kinds of vibration signals. Finally, it can shield the vast majority of interference and only respond to the TPI intrusion.

In order to verify the property of the system, we collected various actions near the oil pipeline by using DAS system which is developed by the authors at the end of fiber about $47 \mathrm{~km}$. The actions include vibration signals generated by digging, walking, running, and vehicles. Due to the test location is close to the roadside, and all kinds of vehicles, especially heavy vehicles, will generate large vibration signal when passing through the pipeline, which may cause false alarms.

Figure 23 shows the amplitude-time-distance relationship of the vibration caused by digging near the test point. Between $47250 \mathrm{~m}$ and $47270 \mathrm{~m}$, the amplitude of the vibration signal is large, which is appeared in the waterfall diagram as bright spots appear along with the excavation action rhythm. The signals at the above spatial positions are superimposed to obtain the vibration time-domain signals, self-power spectrum, and the short-time Fourier transform (STFT) under different intrusion actions as shown in Fig. 24.

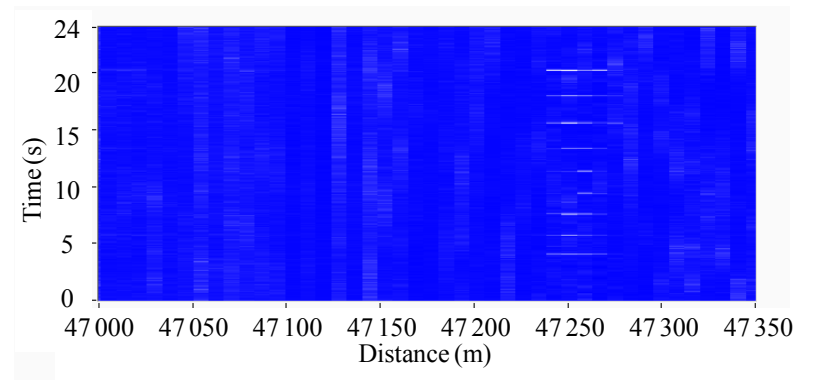

Fig. 23 Waterfall figure of vibration amplitude-timedistance when digging the ground at $1 \mathrm{~m}$ distance away from optical cable.

In addition to the excavation action that the system needs to alarm, in the field, we also collect 
and analyze other common actions that generate vibration (running, jumping, walking, etc.). According to the test results, the pattern characteristics of the various vibration signals are summarized as follows.

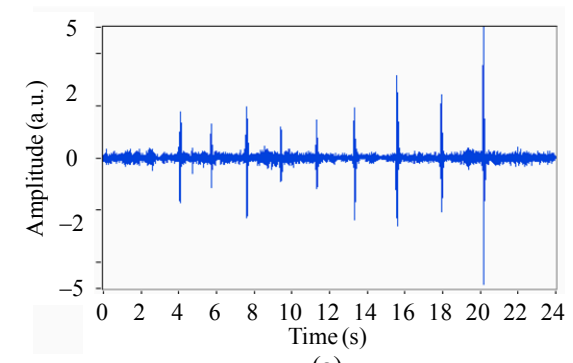

(a)

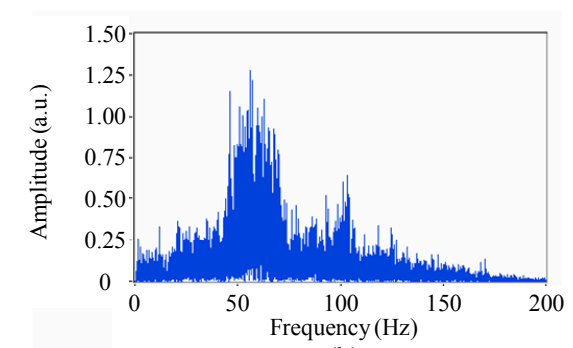

(b)

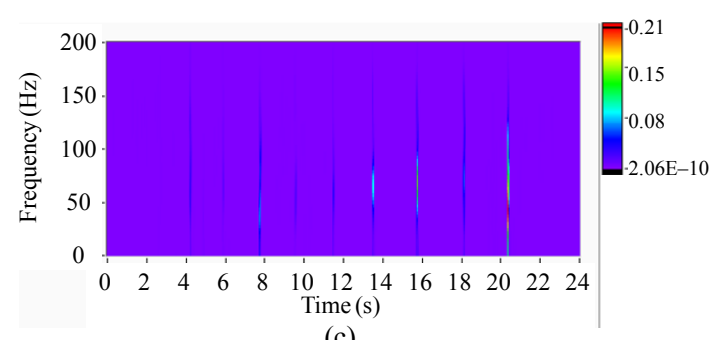

(c)

Fig. 24 (a) Time-domain waveform, (b) frequency spectrum, and (c) STFT when digging the ground at $1 \mathrm{~m}$ vertical distance away from the optical cable.

(1) Time-domain amplitude characteristics: actions such as digging, jumping, and other actions, can produce large vibration signals, while the vibration generated by human walking is much smaller, which can be easily obtained by comparing the time-domain signals.

(2) Time-domain persistence characteristics: the signals generated by vehicles is longer lasts for a long time (on the order of seconds). In Fig. 25, for example, when the train passes, the vibration signal is large, and the duration is very long, forming a continuous vibration wave.

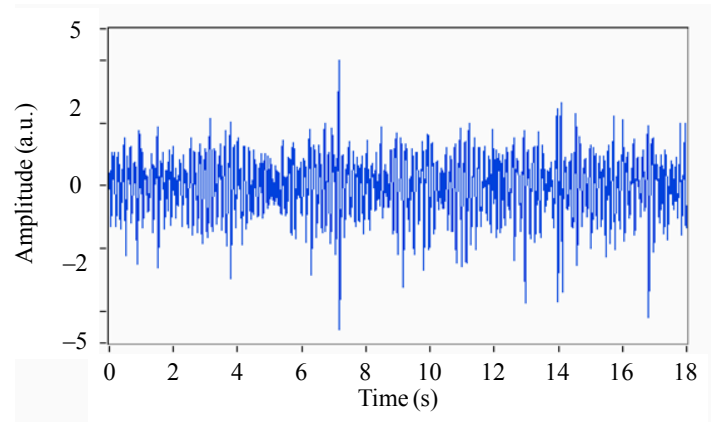

Fig. 25 Time-domain waveform of vibrations generated by the train.

(3) Power spectrum characteristics: the main frequency component of the excavation-generated vibration is within $50 \pm 15 \mathrm{~Hz}$. Compared with signals the human running and jumping generated, there is a large difference in the main frequency components.

(4) Instantaneous frequency characteristics: with the comparison of Figs. 26(a) and 26(b), the energy of the vibration signal generated by excavation is mainly concentrated in the higher frequency of $50 \mathrm{~Hz}-100 \mathrm{~Hz}$, while for the signal generated by jumping, the energy from $0 \mathrm{~Hz}-60 \mathrm{~Hz}$ has a very good continuation.

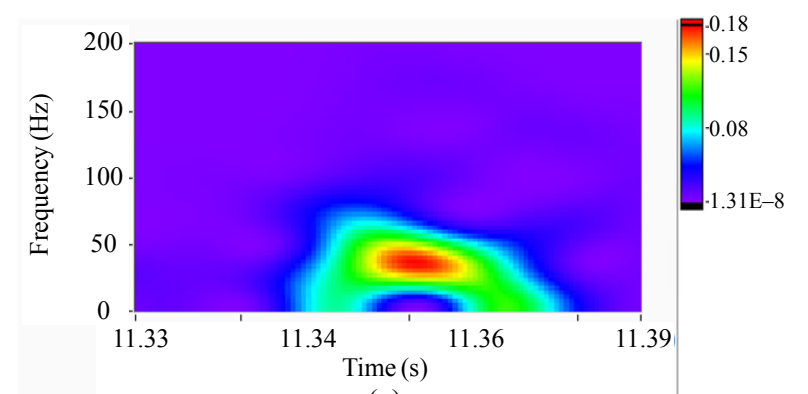

(a)

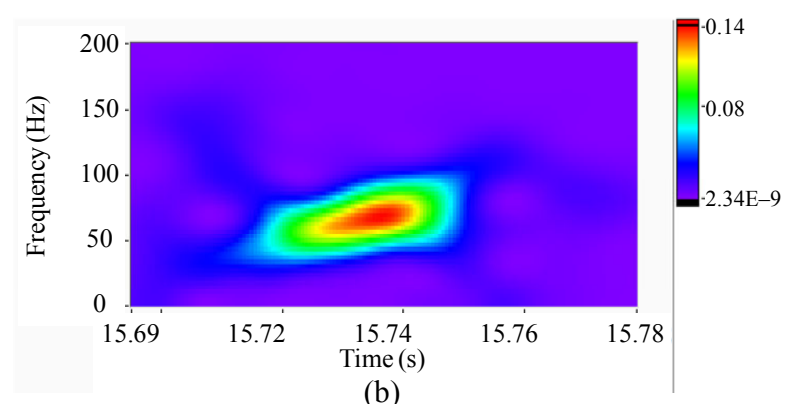

(b)

Fig. 26 STFT figure of (a) digging and (b) running or jumping at $1 \mathrm{~m}$ vertical distance away from the cable.

From the above four signal characteristics, a multi-dimensional criterion is formed, which, on the 
one hand, forms an effective detection of the specific vibration of interests in oil pipeline safety monitoring, such as excavation (digging) action, and on the other hand, the external interference, such as normal human activity and vibration generated by vehicles, can be effectively shielded to reduce system errors and alarms, thus laying a good foundation for the subsequent formation of a truly practical long-range oil pipeline safety monitoring system.

\subsection{Borehole oil/gas exploration with UDAS based on Ф-OTDR}

DAS, with many advantages over conventional geophones, has been used for borehole vertical seismic profiling (VSP) [23, 59], oil/gas exploration $[60,61]$, etc., in recent years. As for traditional borehole seismic acquisition, an array of very expensive geophones is placed inside the well and attached to the wall [62]. After a series of shots from a seismic source are fired and the corresponding data are obtained, such an array of geophones needs to be moved downwards to repeat another seismic acquisition. This procedure will take a quite long time to complete, as the number of geophones is limited. The effective acquisition length of each time is normally less than $300 \mathrm{~m}$, assuming the spatial resolution is $10 \mathrm{~m}$ and the number of geophones is 30. Hence, it would take 10 times to complete whole borehole seismic acquisition for a $3 \mathrm{~km}$-deep well. Nevertheless, DAS can provide much faster deployment/acquisition as it only uses one single optical cable to replace the geophone array, shortening the assembly time and saving the operational costs considerably. Furthermore, DAS can offer much higher spatial resolution as it only depends on the frequency and width of the input optical pulses generated by the modulator. More importantly, optical cables can stand higher temperature and have longer lifetime over electronic geophones. Therefore, DAS is much more suitable, cost-effective and efficient than conventional geophones for borehole seismic acquisition. For traditional ground seismic acquisition, DAS has similar features with borehole seismic acquisition, but a long-distance ground DAS would take huge advantages in terms of improvement in acquisition efficiency and saving in operational costs, if the three components of seismic waves could be detected properly.

The ultra-sensitive distributed acoustic sensing (uDAS) system invented by the authors has been used for both borehole seismic applications. By employing multi-frequency pulse modulation, low-noise optical pulse amplification and self-stabilized $3 \times 3$ phase demodulation, the uDAS system can obtain ultra-high strain resolution of well below $0.1 \mathrm{n} \varepsilon / \sqrt{ } \mathrm{Hz}$ over the frequency range of $4 \mathrm{~Hz}-$ $500 \mathrm{~Hz}$, and reach $\sim 18.2 \mathrm{p \varepsilon} / \sqrt{\mathrm{Hz}}$ at $15 \mathrm{~Hz}$ with spatial resolution of $5 \mathrm{~m}$. Its spatial resolution is adjustable between $2 \mathrm{~m}-5 \mathrm{~m}$ and the dynamic measurement range is $\sim 126 \mathrm{~dB}$.

This uDAS system has been utilized in a series of experiments in oil field test of borehole VSP. In order to compare its performance with conventional geophone array, a dual-well VSP test is arranged as shown in Fig. 27. The geophone array and the uDAS system are placed in adjacent oil wells of A and B, respectively. It can be seen from Fig. 28 that the uDAS system performances are similar to that of conventional geophone arrays, and the data collection efficiency is much higher while the operation cost is much lower. Therefore, it can be proved that uDAS can obtain full-stage, high-density data, coverage times and imaging range, which meets the requirements and has good application prospects in oil/gas exploration.

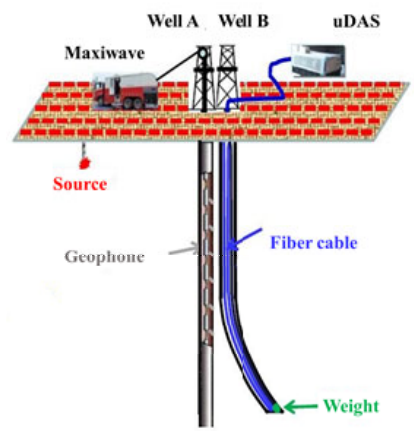

Fig. 27 Schematic diagram of dual-well VSP test. 


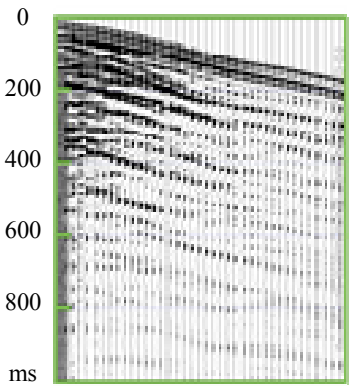

(a)

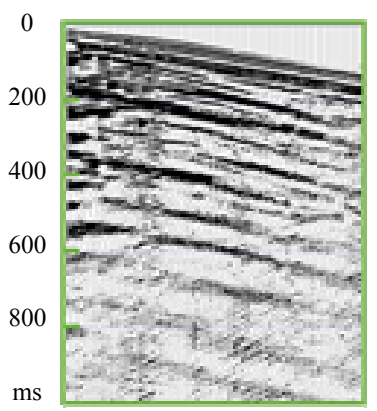

(e)

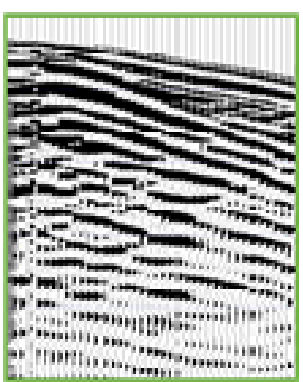

(b)

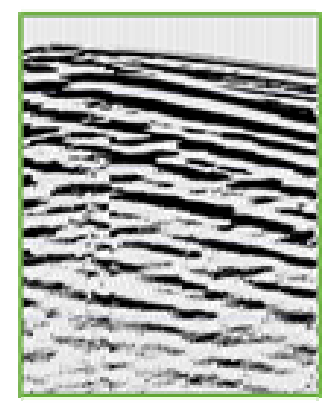

(f)

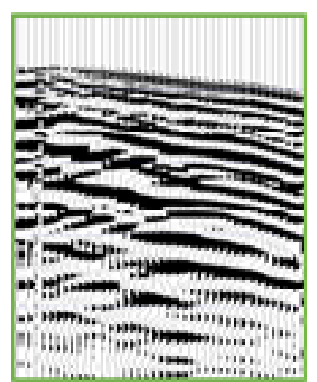

(c)

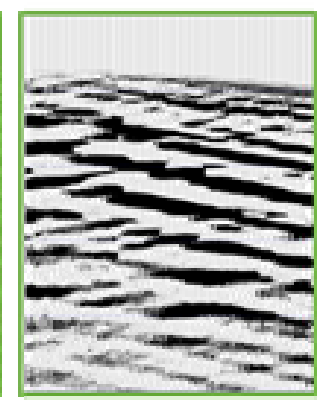

(g)

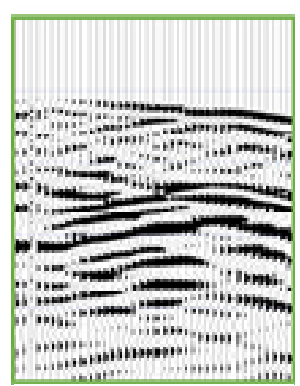

(d)

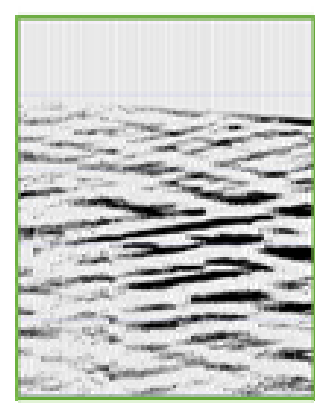

(h)

Fig. 28 Shot point data of geophone array at offsets of (a) $100 \mathrm{~m}$; (b) $305 \mathrm{~m}$; (c) $662 \mathrm{~m}$; (d) $984 \mathrm{~m}$ and the uDAS system at offsets of (e) $100 \mathrm{~m}$; (f) $305 \mathrm{~m}$; (g) $662 \mathrm{~m}$; (h) $984 \mathrm{~m}$.

For the uDAS borehole seismic acquisition system, the distributed optical fiber has high efficiency, high density, high applicability of acquisition work ability, high sensitivity, etc. The sampling interval time and spatial sampling density of uDAS are $0.1 \mathrm{~ms}$ and $0.1 \mathrm{~m}$, respectively, with the high temperature resistance of $250{ }^{\circ} \mathrm{C}$ and high pressure resistance of up to $180 \mathrm{MPa}$. The authors have developed a new method and device for laying optical cable along the oil/gas well, which greatly improves the efficiency and safety of laying optical cable, and effectively solves the coupling problem of optical cable in well. By the end of 2019, the uDAS system has completed 34 times well production tests in 6 oilfields, including Changqing, Xinjiang and Southwest China, and achieved $\sim 7500 \mathrm{~m}$ ultra-deep well operation, significantly improving the observation and imaging capability of the whole well and reducing the construction cost by $\sim 30 \%$ compared to the conventional geophones. It opens a new era for high-precision well-ground joint exploration and reservoir development, and would provide a powerful monitoring tool for oil/gas exploration, storage, and production in complex geo-structures. It is worth to point out that the uDAS system has been elected as one of the ten most significant technology advances of PetroChina in 2109 , which is the highest honor for technological invention in oil/gas industry in China [63].

\subsection{Distributed optical fiber hydrophone with uDAS based on $\Phi$-OTDR}

Compared with traditional electronic hydrophones, optical fiber hydrophone (OFH), which was first demonstrated in 1970s [64, 65], has superior advantages in sensitivity, dynamic range, sensing distance, noise level, frequency response range, size, weight, etc. [66, 67]. It is also immune to the electromagnetic interference and much more cost-effective for large-scale networking. One of the most important applications of $\mathrm{OFH}$ is to detect submarine stratigraphic structures, which is crucial for submarine resource exploration and seismic monitoring, etc.

Distributed OFH based on the DAS technology has unique advantages of longer sensing range, larger array of sensing elements, wider frequency response, lower cost, and longer service life when 
compared with conventional point interferometric OFHs. Liu et al. proposed an OFH based on DAS, with $-148 \mathrm{~dB}$ re $\mathrm{rad} / \mu \mathrm{Pa}$ acoustic pressure sensitivity in 2017 [68]. Then, the acoustic pressure sensitivity was improved to $-141.6 \mathrm{~dB}$ re $\mathrm{rad} / \mu \mathrm{Pa}$ with an elastic sensitization layer [69]. Despite the promise of DAS-based OFH, the sensitivity reported to date is not good enough for practical uses. In 2020, the authors proposed and demonstrated a highly sensitive DAS-based OFH. With improvement in both the interrogation instrument and sensing structure, an average acoustic pressure sensitivity of $-131.0 \mathrm{~dB} \mathrm{re} \mathrm{rad} / \mu \mathrm{Pa}$ was achieved over the frequency range of $1 \mathrm{~Hz}-1024 \mathrm{~Hz}$. We believe that the distributed OFH technology can prove its value in various ocean applications and pave the way to realize a new generation of OFH in the near future.

The experimental setup for sensitivity test of the OFH is shown in Fig. 29(a). In order to improve the sensitivity, the sensing elements of the DAS-based $\mathrm{OFH}$ were fabricated by winding bend-insensitive optical fiber helically on an elastomer. The transducer converts electric signals into acoustic waves, which generates a tiny pressure on the $\mathrm{OFH}$, and then the phase change of the $\mathrm{OFH}$ is demodulated through the uDAS system. The acoustic pressure sensitivity versus different frequency is shown in Fig. 29(b). It can be seen that a flat frequency response is achieved over $1 \mathrm{~Hz}-$ $1024 \mathrm{~Hz}$ and the average acoustic pressure sensitivity level of the $\mathrm{OFH}$ is as high as $-131.0 \mathrm{~dB} \mathrm{re} \mathrm{rad} / \mu \mathrm{Pa}$ with a standard deviation of only $\pm 0.8 \mathrm{dBrerad} / \mu \mathrm{Pa}$.

Then, cabling of the OFH was achieved by winding the specially-designed optical fiber on a continuous elastomer and covering a cable jacket for waterproof and protection, as shown in Fig. 30. The realization of such cabling represents a success of the whole processing craft that makes the long-distance OFH cable available with flexible sensing resolution. It is anticipated that an $\mathrm{OFH}$ cable with 1000 or even 10000 sensing units could be made for distributed underwater acoustic detection over long distance. The large sensing capacity potential, in combination with high sensitivity at low frequency, would make the DAS-based distributed OFH array a killer technology for many ocean applications.

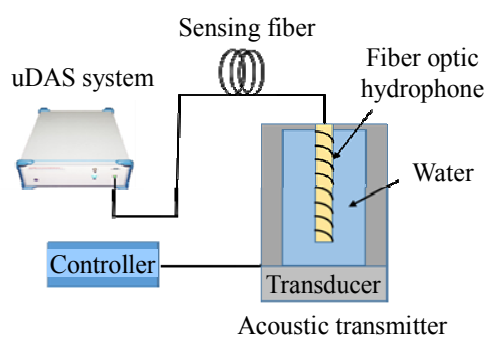

(a)

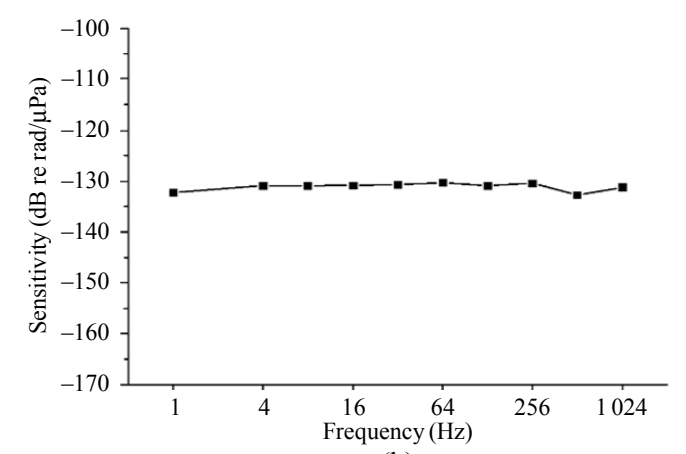

(b)

Fig. 29 (a) Schematic diagram of experimental setup for sensitivity test and (b) calibration curve of acoustic pressure sensitivity level.

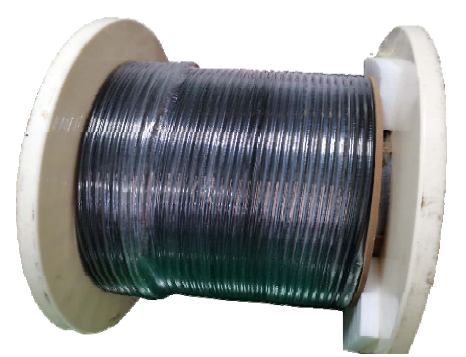

Fig. 30 Optical fiber hydrophone cable for distributed underwater acoustic detection.

\subsection{Applications of artificial intelligence (AI) to Ф-OTDR/DAS}

The $\Phi-O T D R$ technology is still challenging to find more efficient way to achieve highly intelligent and accurate recognition in its signal processing, as vibration or sound signals usually exhibit non-linear and non-stationary characteristics influenced by complicated environments in the real world and geographical conditions of burying sensing cable. 
Researchers have made great efforts to reduce the nuisance alarm rate (NAR) and improve its detection ability for intrusion detection with traditional machine learning and recent AI technologies [70]. At the first stage (before 2015), researches are devoted to achieve improved SNR for reliable detection and location of the events, by post-processing with correlation and moving average [71], 2-dimensional (2-D) image edge operator [72], wavelet transform [73], Hilbert-Huang transform [74], and other signal denoising techniques $[75,76]$. These methods are helpful to depress the noises caused by frequency shift of laser, amplified spontaneous emission noises, and stationary environment noises. However, in real applications, it is found that the NAR mainly comes from poor understanding of the vibration targets especially in complicated time-varying noisy environments. Thus, in the second stage (from 2015 till now), more work is focused on proper feature extraction in various analysis domains and classifier exploration in different ways. The features include magnitude [77], level crossing rate [78], periodic gait characteristics [79] in time domain, energy distribution of fast Fourier transform spectra [80], morphological features in time-space domain [81], the time-frequency spectra obtained by STFT [82], wavelet or wavelet packet decomposition [83], and Mel-frequency cepstral coefficient [84]. The classification models include artificial neural networks [85], support vector machine (SVM) [77] and relevance vector machine [81], etc.

Actually, for a large monitoring range, the $\Phi-O T D R$ signal recognition is quite difficult due to different geological and ground surface conditions as well as complicated environments that may include many kinds of production and life noises. To discriminate threatening vibration sources and various complex time varying background noises correctively, multilevel and multi-orientation feature extraction is the primary work with top priority.
Thus, more and more abundant and comprehensive features are still needed to ensure stable and reliable recognition in real applications. On the other hand, how to make full use of the obtained information is also a valuable work worthy of further study. In 2019, to better utilize the temporal contextual information and especially to further understand the dynamic evolution process of the detected signal and improve its detection ability, a dynamic time sequence recognition and knowledge mining method based on the hidden Markov models (HMMs) is introduced into the DAS system [86]. The processing flow of the HMMs-based methodology is shown in Fig. 31. Local structure feature of the signal is firstly extracted in multiple analysis domains in the time sequence order and then the HMMs are trained, built and used to mine the temporal evolution information and identify the sequential state process of typical events.

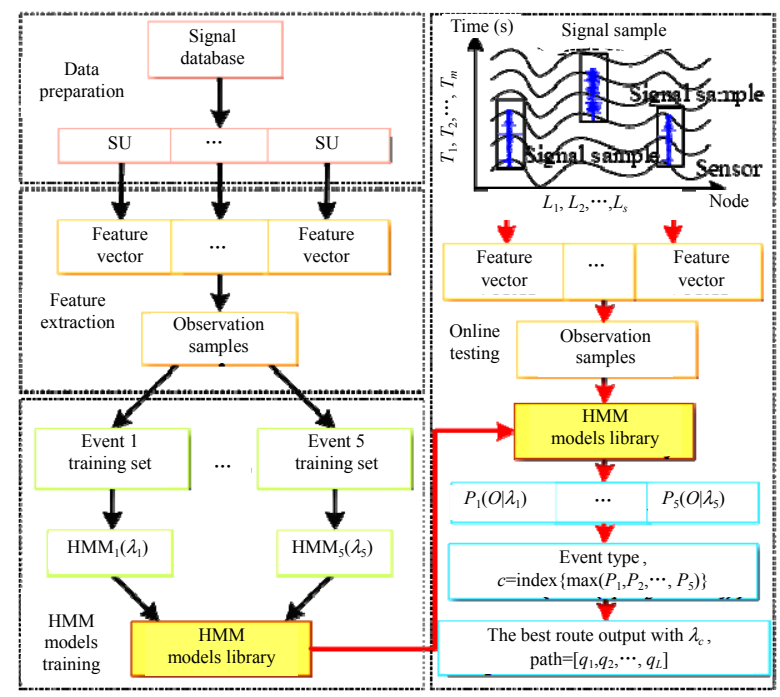

Fig. 31 Processing flow of the HMMs-based methodology.

The database is constructed for five typical events, which are frequently encountered, stationary background noises, manual diggings, mechanical excavations, complicated traffic interferences and factory noises. The typical signals for the five events are demonstrated respectively as in Figs. 32(a) 32(f). And the evolution routes excavated by HMM are illustrated in Fig. 33. It demonstrates that the routes basically coincide but don't exactly behave 
the same with the signal changing trends. It is good for recognition because each route is a statistical result of one type of event signals, which represents the development law of the event. It is more stable than the signal itself and its characteristics, and it

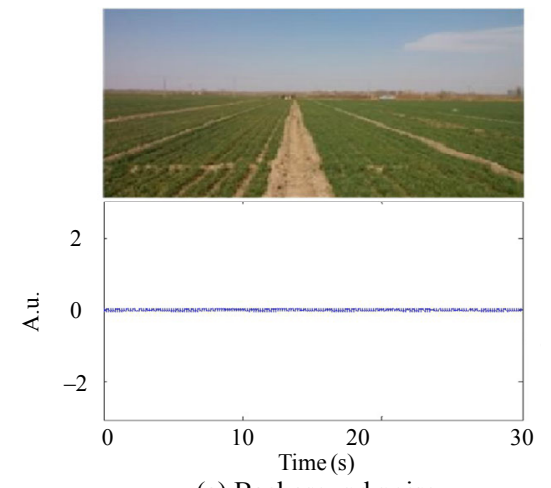

(a) Background noise

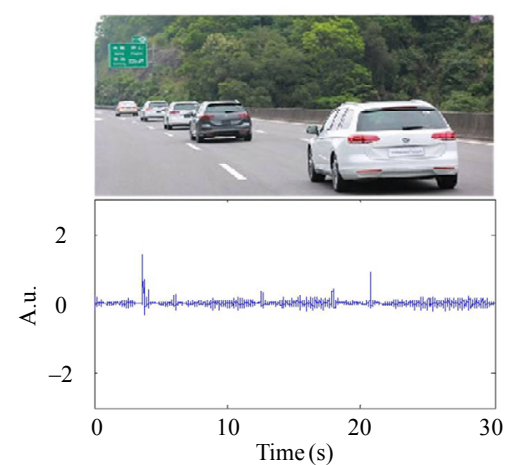

(d) Traffic interference

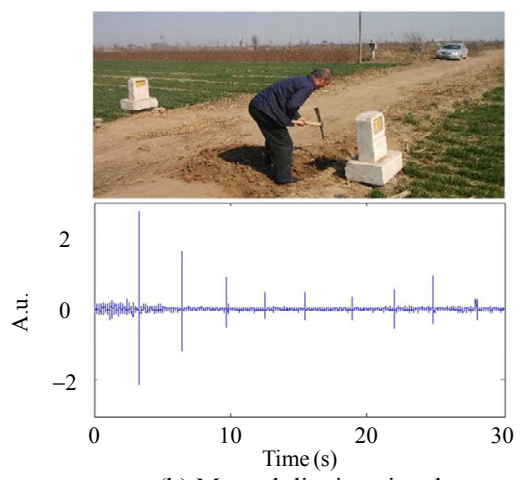

(b) Manual digging signal

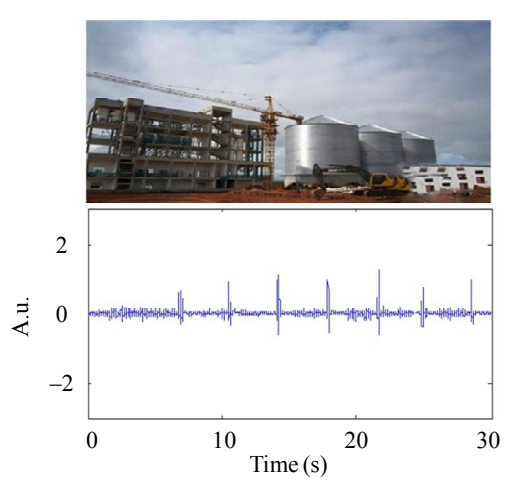

(e) Forging plant noise

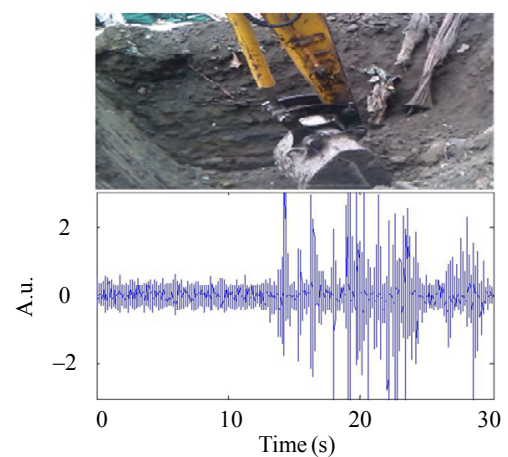

(c) Machine excavation signal

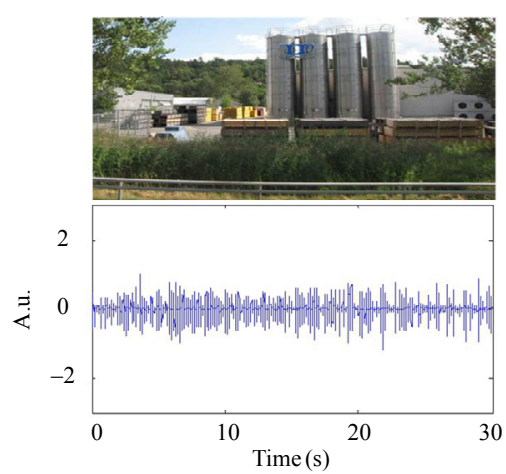

(f) Fabricating plant noise

Fig. 32 Six signal samples for the five target events.

All these methods enhance the perception and recognition ability of DAS further, but they rely heavily on the expert knowledge. Then with rapid development of AI technologies, some researchers began to use 2-D convolution neural networks (CNNs) $[87,88]$ to automatically extract the hidden distinguishable feature of different signal targets, which makes a breakthrough for the feature extraction, and also gets improved recognition results. However, most of the methods still focus on a single level structure feature extracted in a fixed signal frame, which ignore the multi-scale and contextual information of the temporal evolution process. Then J. Tejedor et al. proposed to utilize the temporal contextual information of $\Phi$-OTDR by integrating the tandem feature vectors in a multi-layer perceptron network [89]. In [90], a new multi-scale convolutional neural network was applied to enrich the local structural feature and improve its recognition performance.

In order to further improve the accuracy and efficiency of the vibration signal intelligent recognition, an efficient 1-dimensional (1-D) CNN-SVM network as shown in Fig. 34 is proposed in 2019, which is the key phase to achieve highly real-time intelligent recognition [91]. In order to reduce the impact of noise in the practical application environment on the recognition accuracy, the signal-noise separation method based on a 3-level wavelet packet decomposition is first carried out. And the denoising result is shown in Fig. 35. Five typical events mentioned above are selected as the identification targets to evaluate the proposed method. The 1-D CNN, with three convolutional layers, two full connected (FC) layers, and a softmax layer following the last full connected layer 
FC2, is specially customized for processing the 1-D signals at different fiber locations. Each convolutional layer is followed by a max-pooling layer. Finally, CNN features are extracted automatically with the optimal parameters obtained through the above training process, and prepared for the following classification.

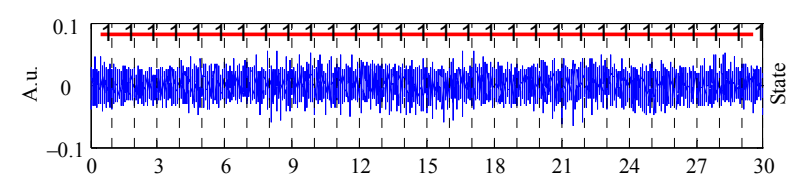

(a) Background noise

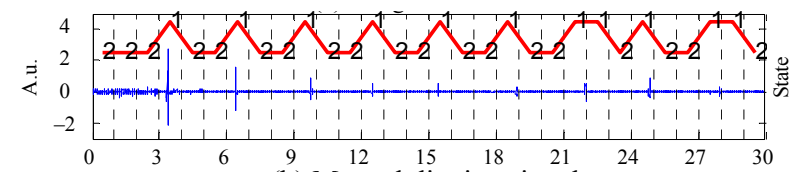

(b) Mannal digging signal

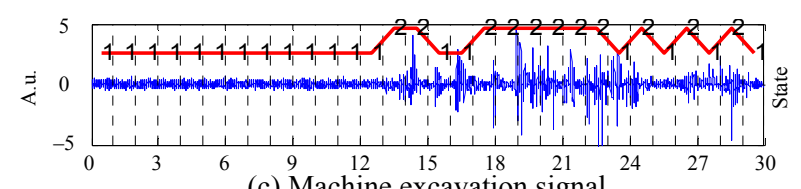

(c) Machine excavation signal

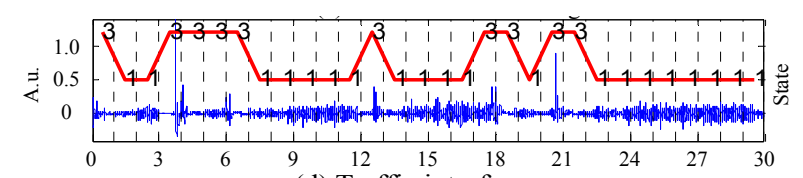

(d) Traffic interference

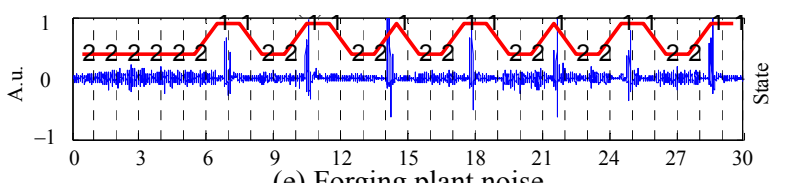

(e) Forging plant noise

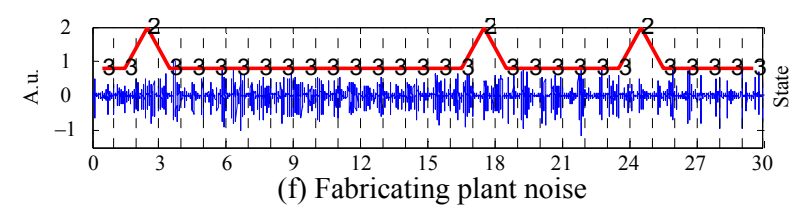

Fig. 33 Evolution routes mined from the HMM models for the six signal samples in Fig. 32.
Table 1 Comparison of recognition results for the six methods.

\begin{tabular}{|c|c|c|c|c|}
\hline Classifier & Event types & Precision & Recall & F-score \\
\hline \multirow{5}{*}{$\begin{array}{c}\text { average } \\
\text { accuracy }=0.982\end{array}$} & 1 & 1.0000 & 1.0000 & 1.0000 \\
\hline & 2 & 1.0000 & 1.0000 & 1.0000 \\
\hline & 3 & 1.0000 & 1.0000 & 1.0000 \\
\hline & 4 & 0.9524 & 1.0000 & 0.9756 \\
\hline & 5 & 1.0000 & 0.9130 & 0.9545 \\
\hline \multirow{5}{*}{$\begin{array}{c}\text { SVM } \\
\text { average } \\
\text { accuracy }=0.919\end{array}$} & 1 & 1.0000 & 1.0000 & 1.0000 \\
\hline & 2 & 0.7500 & 1.0000 & 0.8571 \\
\hline & 3 & 1.0000 & 1.0000 & 1.0000 \\
\hline & 4 & 0.8974 & 0.8750 & 0.8861 \\
\hline & 5 & 1.0000 & 0.8261 & 0.9048 \\
\hline \multirow{5}{*}{$\begin{array}{c}\text { Random forest } \\
\text { average } \\
\text { accuracy }=0.928\end{array}$} & 1 & 1.0000 & 0.9524 & 0.9756 \\
\hline & 2 & 0.8667 & 0.8667 & 0.8667 \\
\hline & 3 & 0.9231 & 1.0000 & 0.9600 \\
\hline & 4 & 0.9000 & 0.9000 & 0.9000 \\
\hline & 5 & 0.9130 & 0.9130 & 0.9130 \\
\hline \multirow{5}{*}{$\begin{array}{l}\text { Xgboost model } \\
\text { average } \\
\text { accuracy }=0.937\end{array}$} & 1 & 0.9524 & 1.0000 & 0.9756 \\
\hline & 2 & 0.8667 & 1.0000 & 0.9286 \\
\hline & 3 & 1.0000 & 1.0000 & 1.0000 \\
\hline & 4 & 0.9750 & 0.8667 & 0.9176 \\
\hline & 5 & 0.8696 & 0.9524 & 0.9091 \\
\hline \multirow{5}{*}{$\begin{array}{c}\text { Decision tree } \\
\text { average } \\
\text { accuracy }=0.892\end{array}$} & 1 & 1.0000 & 0.9524 & 0.9756 \\
\hline & 2 & 0.8125 & 0.8667 & 0.8387 \\
\hline & 3 & 1.0000 & 1.0000 & 1.0000 \\
\hline & 4 & 0.8611 & 0.7750 & 0.8158 \\
\hline & 5 & 0.7407 & 0.8696 & 0.8000 \\
\hline \multirow{5}{*}{$\begin{array}{c}\text { Bayesian network } \\
\text { average } \\
\text { accuracy }=0.783\end{array}$} & 1 & 0.9524 & 1.0000 & 0.9756 \\
\hline & 2 & 0.6667 & 0.4545 & 0.5405 \\
\hline & 3 & 1.0000 & 0.9231 & 0.9600 \\
\hline & 4 & 0.5750 & 0.7931 & 0.6667 \\
\hline & 5 & 0.9565 & 0.8148 & 0.8800 \\
\hline
\end{tabular}

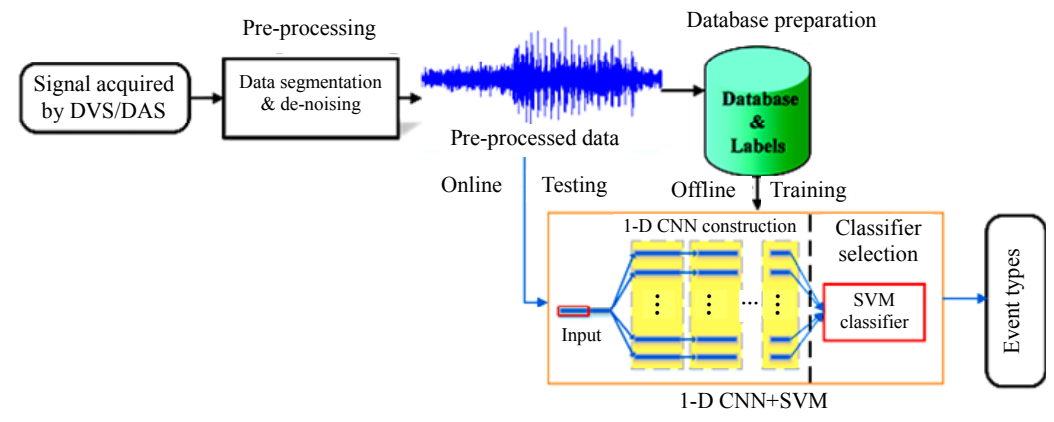

Fig. 34 Proposed 1-D CNN-SVM model.

The proposed method (1-D CNN-SVM) is evaluated by comparing it with the conventional 
machine learning method (Hand-crafted featureSVM). Through the 10-fold-cross validation in Fig. 36, it shows the recognition rate of $96 \%$ can be steadily achieved by the proposed method, which is much better than that of the conventional machine learning method with different groups of data.

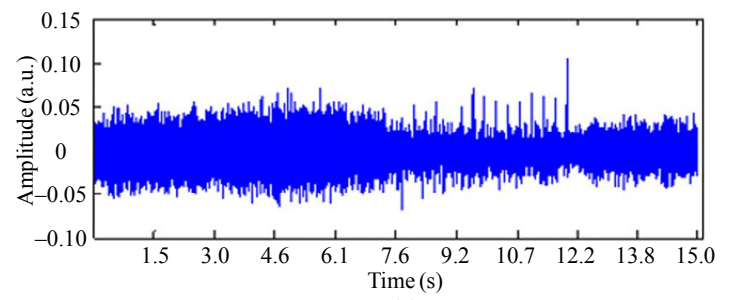

(a)

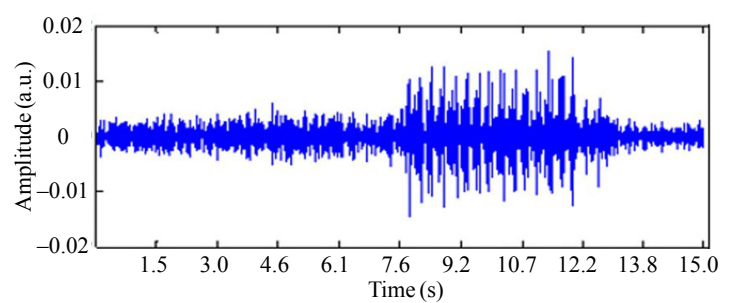

(b)

Fig. 35 Denoising process: (a) the original signal buried in the background and (b) the denoised signal with wavelet packet decomposition.

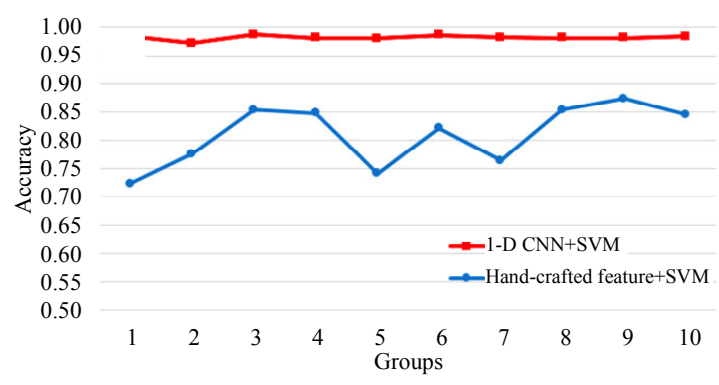

Fig. 36 Ten-fold-cross evaluation for the proposed method and the conventional machine learning method.
Extracting more and more accurate information to understand the detected vibration or acoustic targets better has always been an important goal in signal recognition for DAS. In [92], a novel identification method was then proposed by treating the spatial- and temporal-information in a different way, which is denoted as the 1DCNNs-bidirectional long short term memory (1DCNNs-BiLSTM) model as shown in Fig. 37. Several identical 1D-CNNs are combined in parallel to form a $1 \mathrm{D}-\mathrm{CNN}$ array, in which each 1D-CNN is responsible for the temporal feature extraction for the signal at one spatial node. In the network, in order to alleviate the internal covariate shift phenomenon and increase the feature extracting ability, a batch normalization layer [93] is added following the output of each convolution block. A single layer BiLSTM, including a backward and a forward layer, is designed to extract the spatial connection further among the deep structural feature vectors learned by $\mathrm{CNN}$. In the last step, the FC layer followed by the 1DCNNs-BiLSTM network is used to identify the event type. And a dropout layer is adopted to follow the FC layer to avoid over-fitting phenomenon. From the field test results, it shows the proposed 1DCNNs-BiLSTM is a promising method for promotion in practical complicated environments. It not only digs out the spatiotemporal information of the DAS signals in a deeper level, but also treats it in the two dimensions differently according to the different contribution of them.

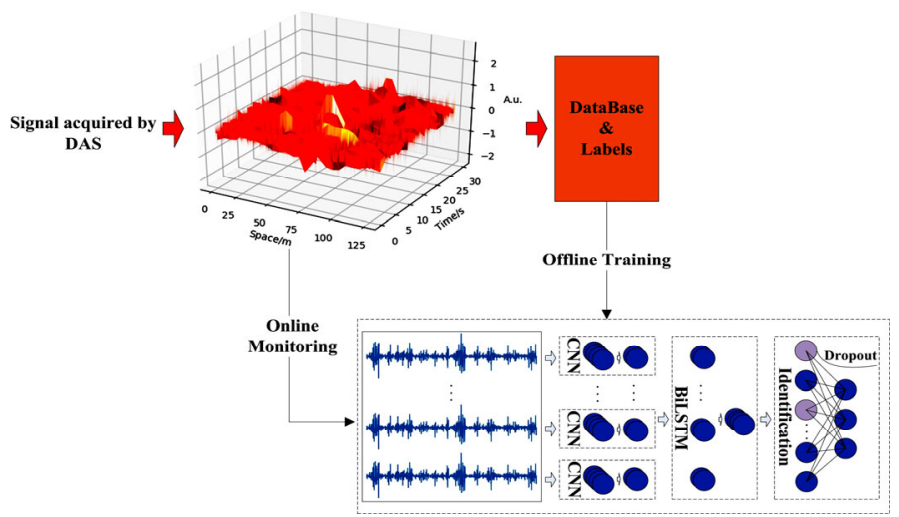

Fig. 37 Proposed 1DCNNs-BiLSTM model.

The classification performance of four methods, namely $1 \mathrm{DCNNs}, 1 \mathrm{DCNNs}-\mathrm{CNN}, 2 \mathrm{D}-\mathrm{CNN}$, and 
1DCNNs-BiLSTM, are compared. As shown in Fig. 38, the proposed 1DCNNs-BiLSTM has the fastest convergence speed of 4 epochs and the highest accuracy of $\sim 97 \%$. Then a ten-fold cross validation is also carried out to verify the stability of the four models and the results are comparatively included in Fig. 36. The results show that the proposed 1DCNNs-BiLSTM always has a better recognition behavior and its average accuracy in the ten tests can be achieved at $97.2 \%$, which is significantly higher than those of the other three methods. It shows that the proposed 1DCNNs-BiLSTM network behaves steadily the best in this field data test for DAS, which generally reveals that the 1DCNNs-BiLSTM has the best learning performance for the spatiotemporal information extraction in this application.

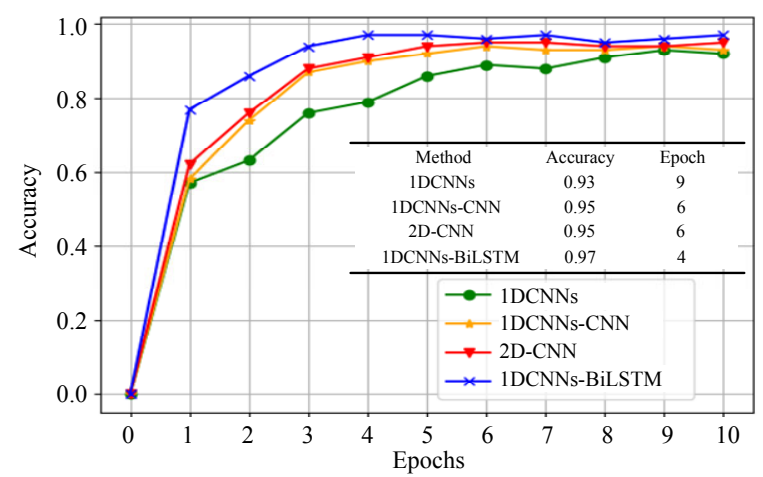

(a)

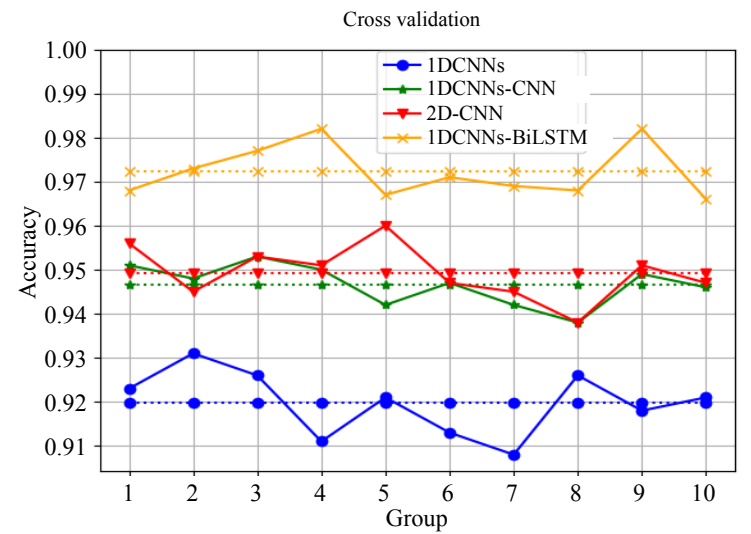

(b)

Fig. 38 Convergence processes of 1DCNNs, 1DCNNs-CNN, 2D-CNN, and 1DCNNs-BiLSTM.

After a review of pattern classification methods with $\mathrm{AI}$ in $\Phi-\mathrm{OTDR}$, we believe that this research direction is still in its early stage. In the future, the main trends of DAS signal recognition are: (1) to extract sufficient information of the sensing signals with multi-level and multi-orientation; (2) to improve the generalization and transfer learning ability of recognition networks; (3) to design comprehensive networks that can complete multi-tasks simultaneously in a single network, such as signal-noise separation, event recognition, and vertical offset-distance location of the target.

\section{Latest progress in key $\Phi$-OTDR components}

\subsection{Low-noise high-order random fiber laser for ultra-long $\Phi-O T D R$}

High-order random Raman fiber lasers (RFLs) have been successfully used as pump sources for DRA with intrinsic modeless property and structural simplicity [94]. Recently, a novel low-noise high-order RFL is demonstrated by the authors, which is common-cavity pumped by an ytterbium-doped random fiber laser (YRFL), where a wavelength division multiplexer (WDM)-assisted fiber-loop mirror is used to generate stable $1090 \mathrm{~nm}$ ytterbium-doped random lasing and cascaded random Raman lasing, simultaneously [95]. The setup of the RFL is shown in Fig. 39.

The output power of the $4^{\text {th }}$-order $1365 \mathrm{~nm}$ random Raman lasing is $8.9 \mathrm{~W}$ with no need of master oscillator power amplification, which can meet the DRA requirement for long-haul fiber-optic transmission systems well. As shown in Fig. 40, with the help of the modeless $1090 \mathrm{~nm}$ YRFL pump, the RIN of such a high-order RFL has been suppressed to $-120 \mathrm{~dB} / \mathrm{Hz}$, which is about $20 \mathrm{~dB}$ lower than that of a commercial RFL pumped by cavity-based ytterbium-doped fiber laser. Therefore, it is anticipated that the sensing distance of $\Phi$-OTDR and DAS systems based on such a novel low-noise high-order DRA pump could be further extended due to the significant reduction in RIN and hence improvement in SNR. 


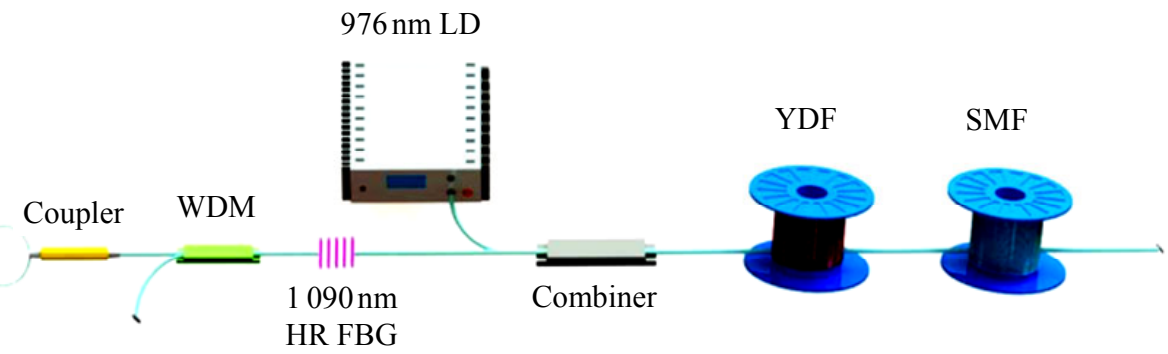

Fig. 39 Experimental setup of the high-order RFL pumped by the YRFL.

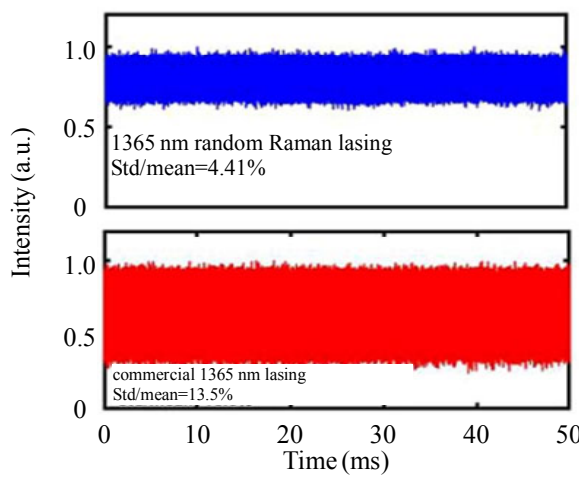

(a)

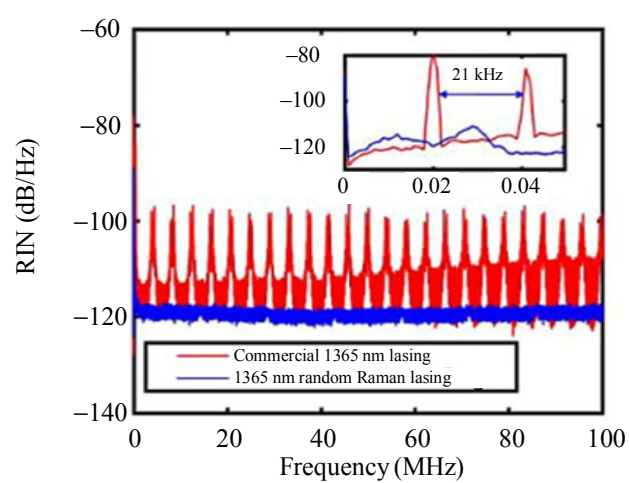

(b)

Fig. 40 (a) Time domain traces of the $1365 \mathrm{~nm}$ random Raman lasing and the commercial $1365 \mathrm{~nm}$ pump and (b) RIN of the $1365 \mathrm{~nm}$ random Raman lasing and commercial $1365 \mathrm{~nm}$ pump. Insert: enlarged view in $0 \mathrm{MHz}-0.05 \mathrm{MHz}$ span. Blue line: $1365 \mathrm{~nm}$ random Raman lasing; red line: commercial $1365 \mathrm{~nm}$ lasing.

\subsection{Cladding softened fiber for sensitivity enhancement of $\Phi$-OTDR}

The $\Phi-O T D R$ technology with high spatial and strain resolutions has been widely used in many practical applications. New sensing fibers that can enhance the phase sensitivity of $\Phi-O T D R$ are worth to be explored, although standard single-mode fiber
(SSMF) has been widely used. Thus, we proposed, fabricated, and tested cladding softened fibers (CSFs) with lower cladding Young's modulus than SSMF, for the first time. The refractive index profile and cross-section photograph of the CSF with $80 \mu \mathrm{m}$ cladding diameter are shown in Fig. 41 [96].

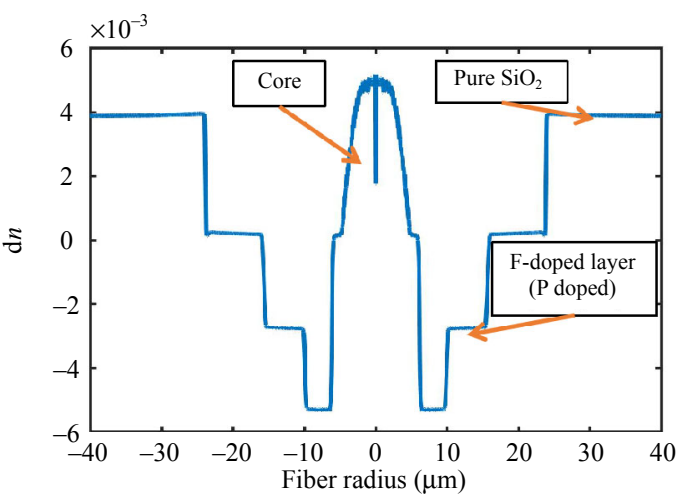

(a)

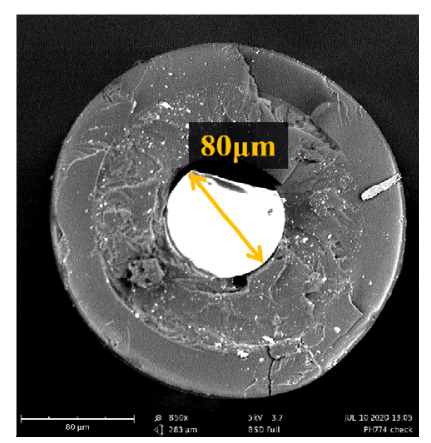

(b)

Fig. 41 (a) Refractive index profile and (b) cross-section photograph of CSF.

The results in Fig. 42 show that the phase sensitivity of the CSF with $0.48 \mathrm{WT} \%$ phosphorusdoping concentration and $80 \mu \mathrm{m}$ cladding diameter is $22 \%$ and $54 \%$ higher than that of the non-phosphorus-doping fiber with $80 \mu \mathrm{m}$ cladding diameter and SSMF, respectively. The linearity between the fiber cladding Young's modulus and 
phase sensitivity improvement is good. It could be predicted that by further increasing the phosphorus-doping concentration to $2 \mathrm{WT} \%$, the fiber phase sensitivity could be enhanced by $\sim 99.5 \%$ over $80 \mu \mathrm{m}$ fiber and $\sim 153.3 \%$ over SSMF correspondingly. In addition, the CSF has a simple preparation process and low cost. Thus, the CSF reported may pave a way for improving the Ф-OTDR phase sensitivity and would be applied to other distributed optical fiber sensing for strain/pressure measurement as a better sensing element over SSMF.

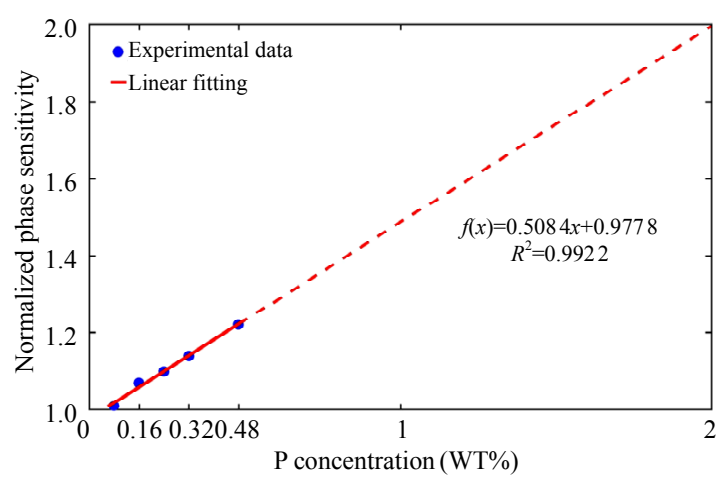

Fig. 42 Normalized phase sensitivity vs phosphorus-doping concentration of CSF.

\subsection{Sensitivity-enhanced optical fiber cable for Ф-OTDR}

In many application cases, $\Phi$-OTDR systems use dark-fiber optical cables directly [97] or specially designed optical cables to obtain additional level of strength enhancement or to withstand extreme temperatures and pressures. Hence, most of these optical cables contain armor reinforcements/ sheaths to assure strength and durability in harsh environments, but this would result in relatively low acoustic sensitivity. In order to improve the acoustic sensitivity, optical cable with helically wound fiber is designed to increase transversal strain sensitivity [98, 99]. Nevertheless, there is little study on sensitivity enhancement of optical cables themselves for $\Phi-O T D R$ applications.

To improve the acoustic sensitivity of the sensing cables in $\Phi-O T D R$, we propose and demonstrate a sensitivity-enhanced optical cable (Fig. 43) with smaller diameter, lighter weight, and greater flexibility than conventional cables [100]. The acoustic sensitivity of the cable has been significantly improved by up to 10 times, when comparing with a rigid cable that has much higher reflection and attenuation of acoustic waves.

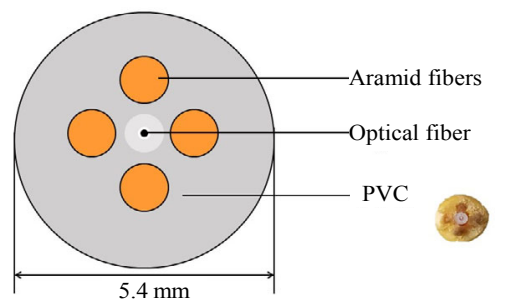

Fig. 43 Structure and cross-sectional photograph of the sensitivity-enhanced cable.

\section{Conclusions}

In recent years, significant advances in $R \& D$ of Ф-OTDR have been made. The performances and applications of $\Phi$-OTDR systems have also been developed rapidly. The $\Phi-O T D R$ technology is capable for sensing vibration and acoustic waves with long detection range, high spatial resolution, and good environment adaptability. In particular with phase demodulation, DAS based on $\Phi$-OTDR has become a powerful tool for accurate detection of vibration and acoustic waves with much better performance than $\Phi-O T D R$. It can be anticipated that the DAS technology would form one of the mainstreams in the field of optical fiber sensors in the near future and may have a revolutionary impact on various fields related to vibration and acoustic detection in complicated environments, such as underground, ocean, and even polar region. In the future, the main R\&D trends of the $\Phi-O T D R / D A S$ technology may be: (1) to achieve ultra-high acoustic strain sensitivity of up to $\mathrm{f} \varepsilon / \sqrt{\mathrm{Hz}}$ order with novel sensing fiber and cable as well as interrogation method for creation of new generation of distributed optical fiber hydrophones/geophones/ sonars as killer technology; (2) to design novel sensing fiber and cable with the ability for 
realization of distributed 3-dimensional (3-D) directional detection of seismic and acoustic waves; (3) to realize ultra-long repeaterless sensing range of up to $200 \mathrm{~km}$ based on ultralow noise DRA for unique use in pipeline/powerline/highway/railway/borderline safety monitoring; (4) to achieve ultra-high spatial resolution of up to $\mathrm{cm}$ order for further improvement of the detection accuracy of acoustic waves; (5) to increase the frequency response range significantly to further expand the application scope of $\Phi-O T D R / D A S$ into the ultrasound region $(\mathrm{MHz}$ order) for nondestructive testing; (6) to construct comprehensive AI networks to be able to complete advanced multi-tasks signal processing simultaneously in a single AI network for achieving better weak signal detection ability and more accurate recognition of weak signals.

In conclusion, the $\Phi-O T D R / D A S$ technology is currently under its rapid development stage and would reach its climax in the next 5 years.

\section{Acknowledgment}

The authors would like to thank all of the members in the FORC at UESTC for their hard work and important contributions to this work. This work was funded by the Natural Science Foundation of China (Grant Nos. 41527805 and 61635005) and the 111 Poject (Grant No. B14039).

Open Access This article is distributed under the terms of the Creative Commons Attribution 4.0 International License (http://creativecommons.org/licenses/by/4.0/), which permits unrestricted use, distribution, and reproduction in any medium, provided you give appropriate credit to the original author(s) and the source, provide a link to the Creative Commons license, and indicate if changes were made.

\section{References}

[1] J. Park, W. Lee, and H. F. Taylor, "Fiber optic intrusion sensor with the configuration of an optical time-domain reflectometer using coherent interference of Rayleigh backscattering," International Society for Optics and Photonics,
China, 1998, 3555: 49-56.

[2] C. D. Butter and G. B. Hocker, "Fiber optics strain gauge," Applied Optics, 1978, 17(18): 2867-2869.

[3] H. F. Taylor and C. Lee, "Apparatus and method for fiber optic intrusion sensing," U.S. Patent 5194847, 1993.

[4] R. Juskaitis, A. M. Mamedov, V. T. Potapov, and S. V. Shatalin, "Interferometry with Rayleigh backscattering in a single-mode optical fiber," Optics Letters, 1994, 19(3): 225-227.

[5] K. N. Choi and H. F. Taylor, "Spectrally stable Er-fiber laser for application in phase-sensitive optical time-domain reflectometry," IEEE Photonics Technology Letters, 2003, 15(3): 386-388.

[6] J. C. Juarez, E. W. Maier, K. N. Choi, and H. F. Taylor, "Distributed fiber-optic intrusion sensor system," Journal of Lightwave Technology, 2005, 23(6): 2081-2087.

[7] J. C. Juarez and H. F. Taylor, "Distributed fiber optic intrusion sensor system for monitoring long perimeters," SPIE, 2005, 5778: 692-703.

[8] J. C. Juarez and H. F. Taylor, "Field test of a distributed fiber-optic intrusion sensor system for long perimeters," Applied Optics, 2007, 46(11): 1968-1971.

[9] K. L. Xie, Y. J. Rao, and Z. L. Ran, "Distributed optical fiber sensing system based of Rayleigh scattering light $\Phi$-OTDR using single-mode fiber laser with high power and narrow linewidth," Acta Optica Sinica, 2008, 28(3): 569-572.

[10] J. Z. Li, Y. J. Rao, Z. L. Ran, et al., "Distributed optical fiber perturbation sensing system combined with $\Phi-O T D R$ and POTDR," Acta Optica Sinica, 2009, 38(5): 1108-1113.

[11] Z. Pan, K. Liang, Q. Ye, H. Cai, R. Qu, and Z. Fang, "Phase-sensitive OTDR system based on digital coherent detection," in Asia Communications and Photonics Conference and Exhibition, China, 2011, pp. 1-6.

[12] A. Masoudi, M. Belal, and T. P. Newson, "A distributed optical fiber dynamic strain sensor based on phase-OTDR," Measurement Science and Technology, 2013, 24(8): 085204.

[13] Z. Pan, Z. Wang, Q. Ye, H. Cai, R. Qu, and Z. Fang, "High sampling rate multi-pulse phase-sensitive OTDR employing frequency division multiplexing," 23rd International Conference on Optical Fiber Sensors, Spain, 2014, 9157: 91576X-1.

[14] Y. Shang, Y. Yang, C. Wang, X. Liu, C. Wang, and G. Peng, "Optical fiber distributed acoustic sensing based on the self-interference of Rayleigh backscattering," Measurement, 2016, 79: 222-227.

[15] Z. Wang, L. Zhang, S. Wang, N. Xue, F. Peng, M Fan, et al., "Coherent $\Phi-O T D R$ based on I/Q demodulation and homodyne detection," Optics Express, 2016, 24(2): 853-858.

[16] S. Wang, X. Fan, Q. Liu, and Z. He, "Distributed 
fiber-optic vibration sensing based on phase extraction from time-gated digital OFDR," Optics Express, 2015, 23(26): 33301-33309.

[17] J. Pastor-Graells, H. F. Martins, A. Garcia-Ruiz, S. Martin-Lopez, and M. Gonzalez-Herraez, "Single-shot distributed temperature and strain tracking using direct detection phase-sensitive OTDR with chirped pulses," Optics Express, 2016, 24(12): 13121-13133.

[18] J. Pastor-Graells, J. Nuno, M. R. Fernandez-Ruiz, A. Garcia-Ruiz, H. F. Martins, S. Martin-Lopez, et al., "Chirped-pulse phase-sensitive reflectometer assisted by first-order Raman amplification," Journal of Lightwave Technology, 2017, 35(21): 4677-4683.

[19] M. R. Fernández-Ruiz, L. Costa, and H. F. Martins, "Distributed acoustic sensing using chirped-pulse phase-sensitive OTDR technology," Sensors, 2019, 19(20): 4368.

[20] D. Chen, Q. Liu, and Z. He, "High-fidelity distributed fiber-optic acoustic sensor with fading noise suppressed and sub-meter spatial resolution," Optics Express, 2018, 26(13): 16138-16146.

[21] J. Xiong, Z. Wang, Y. Wu, H. Wu, and Y. Rao, "Long-distance distributed acoustic sensing utilizing negative frequency band," Optics Express, 2020, 28(24): 35844-35856.

[22] P. Jousset, T. Reinsch, T. Ryberg, H. Blanck, A. Clarke, R. Aghayev, et al., "Dynamic strain determination using fiber-optic cables allows imaging of seismological and structural features," Nature Communications, 2018, 9(1): 2509.

[23] E. F. Williams, M. R. Fernández-Ruiz, R. Magalhaes, R. Vanthillo, Z. Zhan, M. González-Herráez, et al., "Distributed sensing of microseisms and teleseisms with submarine dark fibers," Nature Communication, 2019, 10(1): 5778.

[24] F. Peng, H. Wu, X. H. Jia, Y. J. Rao, Z. N. Wang, and Z. P. Peng, "Ultra-long high-sensitivity $\Phi-O T D R$ for high spatial resolution intrusion detection of pipelines," Optics Express, 2014, 22(11): 13804-13810.

[25] Y. J. Rao, J. Luo, Z. L. Ran, J. F. Yue, X. D. Luo, and Z. Zhou, "Long-distance fiber-optic $\Phi-O T D R$ intrusion sensing system," 20th International Conference on Optical Fibre Sensors, United Kingdom, 2009, 7503: 750310.

[26] Z. Peng, Y. Rao, F. Peng, H. J. Wu, X. H. Jia, and X. Y. Li, "Long distance phase-sensitive optical time-domain reflectometer based on heterodyne detection and forward Raman amplification," Journal of Optoelectronics Laser, 2014, 25(4): 724-729.

[27] H. F. Martins, S. Martín-López, P. Corredera, M. L. Filograno, O. Frazão, and M. Gonzalez-Herráez, "Phase-sensitive optical time domain reflectometer assisted by first-order Raman amplification for distributed vibration sensing over $>100 \mathrm{~km}$," Journal of Lightwave Technology, 2014, 32(8): 1510-1518.

[28] Z. N. Wang, J. J. Zeng, J. Li, M. Q. Fan, H. Wu, F. Peng, et al., "Ultra-long phase-sensitive OTDR with hybrid distributed amplification," Optics Letters, 2014, 39(20): 5866-5869.

[29] J. Li, Z. Wang, L. Zhang, F. Peng, S. Xiao, H. Wu, et al., " $124 \mathrm{~km}$ phase-sensitive OTDR with Brillouin amplification," in 23rd International Conference on Optical Fibre Sensors, Spain, 2014, pp. 91575Z.

[30] X. Jia, Y. Rao, Z. Wang, W. L. Zhang, C. X. Yuan, X. D. Yan, et al., "Distributed Raman amplification using ultra-long fiber laser with a ring cavity: characteristics and sensing application," Optics Express, 2013, 21(18): 21208-21217.

[31] C. Headley and G. P. Agrawal, Raman amplification in fiber optical communication systems, America: Academic Press, 2005.

[32] M. Alahbabi, Y. Cho, and T. Newson, "150-km-range distributed temperature sensor based on coherent detection of spontaneous Brillouin backscatter and in-line Raman amplification," Journal of the Optical Society of America B, 2005, 22(6): 1321-1324.

[33] Y. Rao, S. Feng, Q. Jiang, and Z. Ran, "Ultra-long distance $(300 \mathrm{~km})$ fiber Bragg grating sensor system using hybrid EDF and Raman amplification," in 20th International Conference on Optical Fibre Sensors, United Kingdom, 2009, pp. 75031Q.

[34] Z. N. Wang, J. Li, M. Q. Fan, L. Zhang, F. Peng, H. $\mathrm{Wu}$, et al., "Phase-sensitive optical time-domain reflectometry with Brillouin amplification," Optics Letters, 2014, 39(15): 4313-4316.

[35] Q. Yan, M. Tian, X. Li, Q. Yang, and Y. Xu, "Coherent $\Phi-O T D R$ based on polarization-diversity integrated coherent receiver and heterodyne detection," in 2017 25th Optical Fiber Sensors Conference (OFS), South Korea, 2017, pp. 1032383.

[36] H. Gabai and A. Eyal, "On the sensitivity of distributed acoustic sensing," Optics Letters, 2016, 41(24): 5648-5651.

[37] A. Masoudi and T. P. Newson, "High spatial resolution distributed optical fiber dynamic strain sensor with enhanced frequency and strain resolution," Optics Letters, 2017, 42(2): 290-293.

[38] X. He, S. Xie, F. Liu, S. Cao, L. Gu, X. Zheng, et al., "Multi-event waveform-retrieved distributed optical fiber acoustic sensor using dual-pulse heterodyne phase-sensitive OTDR," Optics Letters, 2017, 42(3): 442-445.

[39] J. Xiong, Z. Wang, Y. Wu, Y. Chen, and Y. Rao, "100 km dynamic strain sensing via CP-ФOTDR," in 2018 Asia Communications and Photonics Conference (ACP), China, 2018, pp. 1-3.

[40] Z. Wang, Y. Fu, and Y. Rao, "Method for distributedly measuring polarization transmission 
matrices of optical fiber and system thereof," U.S. Patent 9841349, 2017.

[41] Z. Wang, Y. Fu, X. Qian, L. Zhang, and Y. Rao, "Proposal for distributed measurement of Müller matrix in optical fibers," in 2016 15th International Conference on Optical Communications and Networks (ICOCN), China, 2016, pp. 1-3.

[42] Y. Chen, Y. Fu, J. Xiong, and Z. Wang, "Distributed fiber birefringence measurement using pulse-compression $\Phi-$ OTDR," Photonic Sensors, DOI. 10.1007/s13320-020-0604-3.

[43] Y. Fu, N. Xue, Z. Wang, B. Zhang, J. Xiong, and Y. Rao, "Impact of I/Q amplitude imbalance on coherent $\Phi$-OTDR," Journal of Lightwave Technology, 2018, 36(4): 1069-1075.

[44] N. Xue, Y. Fu, C. Lu, J. Xiong, L. Yang, and Z. Wang, "Characterization and compensation of phase offset in $\Phi-O T D R$ with heterodyne detection," Journal of Lightwave Technology, 2018, 36(23): 5481-5487.

[45] J. Xiong, L. Xie, and Z. Wang, "Optical pulse compression radar at double repetition rate with both positive and negative beat frequencies," in 2017 International Topical Meeting on Microwave Photonics (MWP), China, 2017, pp. 1-4.

[46] Z. Wang, J. Xiong, L. Xie, and Y. Rao, "Method of improving measurement speed of distributed optical fiber sensor by adopting orthogonal signals and system thereof," U.S. Patent 10564012B2, 2020.

[47] Z. Wang, J. Jiang, Z. Wang, J. Xiong, and Y. J. Rao, "Bandwidth-enhanced quasi-distributed acoustic sensing with interleaved chirped pulses," IEEE Sensors Journal, 2020, 20(21): 12739-12743.

[48] J. Xiong, J. Jiang, Y. Wu, Y. Chen, L. Xie, Y. Fu, et al., "Chirped-pulse coherent-OTDR with predistortion," Journal of Optics, 2018, 20(3): 034001.

[49] Z. Wang, B. Zhang, J. Xiong, Y. Fu, S. Lin, J. Jiang, et al., "Distributed acoustic sensing based on pulse-coding phase-sensitive OTDR," IEEE Internet of Things Journal, 2018, 6(4): 6117-6124.

[50] Y. Wu, Z. Wang, J. Xiong, J. Jiang, and Y. Rao, "Bipolar-coding $\Phi-O T D R$ with interference fading elimination and frequency drift compensation," Journal of Lightwave Technology, 2020, 38(21): 6121-6128.

[51] J. Jiang, Z. Wang, Z. Wang, Y. Wu, S. Lin, J. Xiong, et al., "Coherent Kramers-Kronig receiver for Ф-OTDR," Journal of Lightwave Technology, 2019, 37(18): 4799-4807.

[52] Y. Wu, Z. Wang, J. Xiong, J. Jiang, S. Lin, and Y. Chen, "Interference fading elimination with single rectangular pulse in $\Phi$-OTDR," Journal of Lightwave Technology, 2019, 37(13): 3381-3387.

[53] S. Lin, Z. Wang, J. Xiong, Y. Fu, J. Jiang, Y. Wu, et al., "Rayleigh fading suppression in one-dimensional optical scatters," IEEE Access,
2019, 7: 17125-17132.

[54] J. Xiong, Z. Wang, Y. Wu, and Y. Rao, "Single-shot COTDR using sub-chirped-pulse extraction algorithm for distributed strain sensing," Journal of Lightwave Technology, 2020, 38(7): 2028-2036.

[55] R. D. Pascoe and T. N. Eichorn, "What is communication-based train control?" IEEE Vehicular Technology Magazine, 2009, 4(4): 16-21.

[56] P. Ripamonti, "Circuit for detecting unbalance of the traction current in a track circuit," U.S. Patent 4432517, 1984.

[57] F. Peng, N. Duan, Y. Rao, and J. Li, "Real-time position and speed monitoring of trains using phase-sensitive OTDR," IEEE Photonics Technology Letters, 2014, 26(20): 2055-2057.

[58] Y. Zhan, "The harm and countermeasures of soil oil pollution in China," Environmental Pollution and Control, 2008, 30(3): 91-93.

[59] A. Egorov, J. Correa, A. Bóna, R. Pevzner, K. Tertyshnikov, S. Glubokovskikh, et al., "Elastic full-waveform inversion of vertical seismic profile data acquired with distributed acoustic sensors," Geophysics, 2018, 83(3): R273-R281.

[60] K. N. Madsen, S. Dümmong, A. Kritski, D. Finfer, P.N. Travis, T. Bostick, et al., "Simultaneous multiwell VSP using distributed acoustic sensing," in Proceedings of Second EAGE Workshop on Permanent Reservoir Monitoring, Malta, 2013.

[61] J. Ni, C. Wang, Y. Shang, X. Zhang, and Y. Zhao, "Distributed fiber-optic acoustic sensing for petroleum geology exploration," Journal of Physics: Conference Series, 2018, 1065(65): 252029.

[62] A. Mateeva, J. Lopez, H. Potters, J. Mestayer, B. Cox, D. Kiyashchenko, et al., "Distributed acoustic sensing for reservoir monitoring with vertical seismic profiling," Geophysical Prospecting, 2014, 62(4): 679-692.

[63] http://www.cnpc.com.cn/cnpc/sdkjjz/202001/583651 6fb34a48e590ebff3caf0afdd6.shtml.

[64] J. Cole, R. Johnson, and P. Bhuta, "Fiber-optic detection of sound," The Journal of the Acoustical Society of America, 1977, 62(5): 1136-1138.

[65] J. A. Bucaro, H. D. Dardy, and E. F. Carome, "Optical fiber acoustic sensor," Applied Optics, 1977, 16(7): 1761-1762.

[66] D. J. Hill, P. J. Nash, D. A. Jackson, D. J. Webb, S. F. O'Neill, I. Bennion, et al., "Fiber laser hydrophone array," in Fiber Optic Sensor Technology and Applications, United States, 1999, pp. 55-66.

[67] C. K. Kirkendall and A. Dandridge, "Overview of high performance fibre-optic sensing," Journal of Physics D: Applied Physics, 2004, 37(18): R197.

[68] X. Liu, C. Wang, Y. Shang, C. Wang, W. Zhao, G. Peng, et al., "Distributed acoustic sensing with Michelson interferometer demodulation," Photonic Sensors, 2017, 7(3): 193-198.

[69] Y. Yang, T. Xu, S. Feng, J. Huang, and F. Li, 
"Optical fiber hydrophone based on distributed acoustic sensing," in Fiber Optic Sensing and Optical Communication, China, 2018, pp. 108490B.

[70] L. Y. Shao, S. Liu, S. Bandyopadhyay, F. Yu, W. Xu, C. Wang, et al., "Data-driven distributed optical vibration sensors: a review," IEEE Sensors Journal, 2019, 20(12): 6224-6239.

[71] Y. Lu, T. Zhu, L. Chen, and X. Bao, "Distributed vibration sensor based on coherent detection of phase-OTDR," Journal of lightwave Technology, 2010, 28(22): 3243-3249.

[72] T. Zhu, X. Xiao, Q. He, and D. Diao, "Enhancement of SNR and spatial resolution in phi-OTDR system by using two-dimensional edge detection method," Journal of Lightwave Technology, 2013, 31(17): 2851-2856.

[73] Z. Qin, L. Chen, and X. Bao, "Wavelet denoising method for improving detection performance of distributed vibration sensor," IEEE Photonics Technology Letters, 2012, 24(7): 542-544.

[74] X. Hui, S. Zheng, J. Zhou, H. Chi, X. Jin, and X. Zhang, "Hilbert-Huang transform time-frequency analysis in phi-OTDR distributed sensor," IEEE Photonics Technology Letters, 2014, 26(23): 2403-2406.

[75] H. Wu, J. Wang, X. Wu, and Y. Wu, "Real intrusion detection for distributed fiber fence in practical strong fluctuated noisy backgrounds," Sensor Letters, 2012, 10(7): 1557-1561.

[76] H. Wu, S. Xiao, X. Li, Z. Wang, J. Xu, and Y. Rao, "Separation and determination of the disturbing signals in phase-sensitive optical time domain reflectometry(Ф-OTDR)," Journal of Lightwave Technology, 2015, 33(15): 3156-3162.

[77] D. Tan, X. Tian, W. Sun, Y. Zhou, L. Liu, Y. Ma, et al., "An oil and gas pipeline pre-warning system based on $\Phi-O T D R, " ~ O F S 2014$ 23rd International Conference on Optical Fiber Sensors, Spain, 2014, 9157: 91578W.

[78] H. Zhu, C. Pan, and X. Sun, "Vibration waveform reproduction and location of OTDR based distributed optical-fiber vibration sensing system," SPIE, 2013, 9062: 89931P.

[79] N. Fang, L. Wang, D. Jia, C. Shan, and Z. Huang, "Walking intrusion signal recognition method for fiber fence system," 2009 Asia Communications and Photonics conference and Exhibition (ACP), China, 2010, 52: 2381-2384.

[80] Z. Wang, Z. Pan, Q. Ye, H. Cai, and Z. Fang, "Fast pattern recognition based on frequency spectrum analysis used for intrusion alarming in optical fiber fence," Chinese Journal of Lasers, 2015, 42(4): 0405010 .

[81] Q. Sun, H. Feng, X. Yan, and Z. Zeng, "Recognition of a phase-sensitivity OTDR sensing system based on morphologic feature extraction," Sensors, 2015,
15(7): 15179-15197.

[82] H. F. Martins, D. Piote, J. Tejedor, J. Macias-Guarasa, J. Pastor-Graells, S. Martin-Lopez, et al., "Early detection of pipeline integrity threats using a smart fiber optic surveillance system: the PIT-STOP project," International Conference on Optical Fibre Sensors (OFS24), Brazil, 2015, 9634: 96347X.

[83] H. Wu, Y. Qian, W. Zhang, and C. Tang, "Feature extraction and identification in distributed optical-fiber vibration sensing system for oil pipeline safety monitoring," Photonic Sensors, 2017, 7(4): 305-310.

[84] A. K. Fedorov, M. N. Anufriev, A. A. Zhirnov, K. V. Stepanov, E. T. Nesterov, D. E. Namiot, et al., "Note: Gaussian mixture model for event recognition in optical time-domain reflectometry based sensing systems," Review of Scientific Instruments, 2016, 87(3): 036107.

[85] Q. Tian, C. Zhao, Y. Zhang, and H. Qu, "Intrusion signal recognition in OFPS under multi-level wavelet decomposition based on RVFL neural network," Optik, 2017, 146: 38-50.

[86] H. Wu, X. Liu, Y. Xiao, and Y. Rao, "A dynamic time sequence recognition and knowledge mining method based on the hidden Markov models (HMMS) for pipeline safety monitoring with (-OTDR," Journal of Lightwave Technology, 2019, 37(19): 4991-5000.

[87] L. Wen, X. Li, L. Gao, and Y. Zhang, "A new convolutional neural network-based data-driven fault diagnosis method," IEEE Transactions on Industrial Electronics, 2017, 65(7): 5990-5998.

[88] T. Ince, S. Kiranyaz, L. Eren, M. Askar, and M. Gabbouj, "Real-time motor fault detection by 1-D convolutional neural networks," IEEE Transactions on Industrial Electronics, 2016, 63(11): 7067-7075.

[89] J. Tejedor, J. Macias-Guarasa, H. Martins, S. Martin-Lopez, and M. Gonzalez-Herraez, "A contextual GMM-HMM smart fiber optic surveillance system for pipeline integrity threat detection," Journal of Lightwave Technology, 2019, 37(18): 4514-4522.

[90] J. Wu, L. Y. Guan, M. Bao, Y. Xu, and W. Ye, "Vibration events recognition of optical fiber based on multi-scale 1-D CNN," Opto-Electronic Engineering, 2019, 46(05): 180493.

[91] H. Wu, J. Chen, X. Liu, Y. Xiao, M. Wang, Y. Zheng, et al., "One-dimensional CNN-based intelligent recognition of vibrations in pipeline monitoring with DAS," Journal of Lightwave Technology, 2019, 37(17): 4359-4366.

[92] H. Wu, M. Yang, S. Yang, H. Lu, C. Wang, and Y. Rao, "A novel DAS signal recognition method based on spatiotemporal information extraction with 1DCNNs-BiLSTM network," IEEE Access, 2020, 8: 119448-119457. 
[93] S. Ioffe and C. Christian, "Batch normalization: accelerating deep network training by reducing internal covariate shift," IEEE Conference on Computer Vision and Pattern Recognition, Boston, 2015, pp. 03167.

[94] S. K. Turitsyn, S. A. Babin, A. E. El-Taher, P. Harper, D. V. Churkin, S. I. Kablukov, et al., "Random distributed feedback fibre laser," Nature Photonics, 2010, 4(4): 231-235.

[95] B. Han, Y. Rao, H. Wu, J. Yao, H. Guan, R. Ma, et al., "Low-noise high-order Raman fiber laser pumped by random lasing," Optics Letters, 2020, 45(20): 5804-5807.

[96] Y. Rao, X. Jiang, B. Han, et al., "An acoustic sensitization fiber based on cladding softening and multi-cladding structure," C.N. Patent 110045457 ,
2020.

[97] N. J. Lindsey, T. C. Dawe, and J. B. Ajo-Franklin, "Illuminating seafloor faults and ocean dynamics with dark fiber distributed acoustic sensing," Science, 2019, 366(6469): 1103-1107.

[98] B. N. Kuvshinov, "Interaction of helically wound fibre-optic cables with plane seismic waves," Geophysical Prospecting, 2016, 64(3): 671-688.

[99] J. C. Hornman, "Field trial of seismic recording using distributed acoustic sensing with broadside sensitive fibre-optic cables," Geophysical Prospecting, 2016, 65(1): 35-46.

[100] B. Han, H. Guan, J. Yao, et al., "Distributed acoustic sensing with sensitivity-enhanced optical cable," IEEE Sensors Journal, 2020, DOI: 10.1109/JSEN.2020.3035002. 